\title{
Equivalence of Order and Algebraic Properties in Ordered *-Algebras
}

\author{
Matthias Schötz * \\ Département de Mathématiques \\ Université libre de Bruxelles
}

June 2020

\begin{abstract}
The aim of this article is to describe a class of ${ }^{*}$-algebras that allows to treat well-behaved algebras of unbounded operators independently of a representation. To this end, Archimedean ordered ${ }^{*}$-algebras ( ${ }^{*}$-algebras whose real linear subspace of Hermitian elements are an Archimedean ordered vector space with rather weak compatibilities with the algebraic structure) are examined. The order induces a translation-invariant uniform metric which comes from a $C^{*}$-norm in the bounded case. It will then be shown that uniformly complete Archimedean ordered *-algebras have good order properties (like existence of infima, suprema or absolute values) if and only if they have good algebraic properties (like existence of inverses or square roots). This suggests the definition of $S u^{*}$-algebras as uniformly complete Archimedean ordered ${ }^{*}$-algebras which have all these equivalent properties. All methods used are completely elementary and do not require any representation theory and not even any assumptions of boundedness, so $S u^{*}$-algebras generalize some important properties of $C^{*}$-algebras to algebras of unbounded operators. Similarly, they generalize some properties of $\Phi$-algebras (certain lattice-ordered commutative real algebras) to non-commutative ordered *-algebras. It is also shown that the order on $S u^{*}$-algebra is uniquely determined, so $S u^{*}$-algebras are indeed just a class of well-behaved ${ }^{*}$-algebras. As an example, $S u^{*}$-algebras of unbounded operators on a Hilbert space are constructed. They arise e.g. as ${ }^{*}$-algebras of symmetries of a self-adjoint (not necessarily bounded) Hamiltonian operator of a quantum mechanical system.
\end{abstract}

\section{Introduction}

Many important examples of *-algebras, especially *-algebras of complex-valued functions or *-algebras of adjointable endomorphisms, carry a partial order on their Hermitian elements that is compatible with the algebraic structure: In the former case, this is the order by pointwise comparison of realvalued functions, in the latter it is the usual order on Hermitian operators. From a more abstract point of view, it has long been known that there exists an intrinsic partial order on the Hermitian elements of a $C^{*}$-algebra, which can be defined in many equivalent ways (e.g. by declaring squares

${ }^{*}$ Boursier de l'ULB, Matthias.Schotz@ulb.ac.be. This work was supported by the Fonds de la Recherche Scientifique (FNRS) and the Fonds Wetenschappelijk Onderzoek - Vlaaderen (FWO) under EOS Project $\mathrm{n}^{0} 30950721$. 
of Hermitian elements to be the positive ones, or elements with non-negative real spectrum). This is of course not surprising as $C^{*}$-algebras can always be represented as *-algebras of bounded operators, and in the commutative case even as *-algebras of continuous functions. However, a generalization of this approach seems to be difficult, at least in the realm of topological *-algebras: Already Banach *-algebras can have extremely pathological order properties. Because of this, examining *-algebras having in some sense "unbounded" elements by means of locally convex *-algebras is a rather hard task.

However, in the commutative case, ${ }^{*}$-algebras are just the complexifications of real associative algebras. So the theory of ordered real algebras, especially of lattice ordered ones like (almost) $f$-algebras and $\Phi$-algebras, immediately carries over and yields examples of well-behaved ordered *-algebras even beyond the scope of $C^{*}$-algebras. The representation theorem [5, Thm. 2.3] for $\Phi$-algebras as algebras of functions on a compact Hausdorff space with values in the extended real numbers further exemplifies the close relation between commutative $C^{*}$-algebras and (complexifications of) $\Phi$-algebras.

The aim of the present article is to examine ordered ${ }^{*}$-algebras and ultimately to determine a class of very well-behaved ordered *-algebras that generalize important properties of $C^{*}$-algebras to the unbounded case, as well as properties of $\Phi$-algebras to the non-commutative case. This includes the existence of suprema and infima of finitely many commuting Hermitian elements, of absolute values, square roots of positive elements and inverses of elements that are coercive (i.e. "strictly" positive), as well as automatic continuity of unital *-homomorphisms and the uniqueness of the order. Special attention is given to situations where order-theoretic and algebraic concepts are equivalent. The most obvious example for this are absolute values: The absolute value $|a|$ of a Hermitian element $a$ should be, from the purely order-theoretic point of view, the supremum of $a$ and $-a$. But from a more algebraic point of view, $|a|$ should be the (positive) square root of $a^{2}$. This raises the question whether, or under which circumstances, the two descriptions are equivalent.

It will be shown that every Archimedean ordered *-algebra carries a metrizable, translation-invariant topology. In the bounded case, this topology comes from a $C^{*}$-norm as in [10], but there is no need for restriction to this special case: Theorem 7.1 shows that for complete Archimedean ordered *algebras, the first properties mentioned above (from existence of suprema and infima to existence of inverses) are all equivalent and then imply the others (automatic continuity, uniqueness of the order and compatibility or equivalence of some further order-theoretic and algebraic concepts). Those algebras where these equivalent properties are fulfilled will be called $S u^{*}$-algebras. They include $C^{*}$-algebras as well as (complexifications of) complete $\Phi$-algebras as special cases. In the end, examples of $S u^{*}$-algebras of unbounded operators, which are neither $C^{*}$ - nor $\Phi$-algebras, will be constructed. This way, $S u^{*}$-algebras allow to examine ${ }^{*}$-algebras of unbounded operators independently of concrete representations.

The article is organized as follows: The next Section 2 explains the notation and gives some basic and well-known facts especially about ordered vector spaces. Section 3 contains the definition of (quasi-) ordered *-algebras as well as some important examples, both well-behaved and ill-behaved ones, and discusses the construction of the uniform metric. After that, Section 4 describes radical ordered *-algebras which fulfil an additional compatibility between multiplication and order, and it is shown that especially all symmetric ordered *-algebras (those in which "strictly" positive elements have a multiplicative inverse) are radical. The operations $\vee$ and $\wedge$, which describe especially well-behaved 
suprema and infima of two commuting Hermitian elements, are discussed in Section 5. This leads to the definition of $\Phi^{*}$-algebras which are essentially non-commutative generalizations of $\Phi$-algebras. Square roots, which allow the construction of absolute values and thus of suprema and infima, are examined in Section 6. All this then leads to the main Theorem 7.1 in Section 7, which essentially states that in the uniformly complete case, the existence of suprema, infima, absolute values, square roots and inverses are equivalent, and motivates Definition 7.2 of $S u^{*}$-algebras as those complete ordered ${ }^{*}$-algebras where these equivalent conditions are fulfilled. Moreover, all the results obtained in the previous sections (like uniqueness of the order or automatic continuity of unital *-homomorphisms) then apply especially to these $S u^{*}$-algebras. Finally, in Section 8, examples of $S u^{*}$-algebras of unbounded operators on a Hilbert space are constructed.

\section{Preliminaries}

The natural numbers are $\mathbb{N}=\{1,2,3, \ldots\}, \mathbb{N}_{0}:=\mathbb{N} \cup\{0\}$ and the sets of real and complex numbers are denoted by $\mathbb{R}$ and $\mathbb{C}$, respectively. If $X$ is a set, then $\operatorname{id}_{X}: X \rightarrow X$ is $x \mapsto \operatorname{id}_{X}(x):=x$. A quasi-order on $X$ is a reflexive and transitive relation, hence a partial order is a quasi-order that is additionally anti-symmetric. If $X$ and $Y$ are both endowed with a quasi-order $\lesssim$, then a map $\Psi: X \rightarrow Y$ is called $\underline{\text { increasing }}$ if $\Psi(x) \lesssim \Psi(\tilde{x})$ for all $x, \tilde{x} \in X$ with $x \lesssim \tilde{x}$. If $\Psi$ is injective and increasing and if conversely also $x \lesssim \tilde{x}$ holds for all $x, \tilde{x} \in X$ with $\Psi(x) \lesssim \Psi(\tilde{x})$, then $\Psi$ is called an order embedding.

A quasi-ordered vector space is a real vector space $V$ endowed with a quasi-order $\lesssim$ such that $u+w \lesssim v+w$ and $\lambda u \lesssim \lambda v$ hold for all $u, v, w \in V$ with $u \lesssim v$ and all $\lambda \in[0, \infty[$. An ordered vector

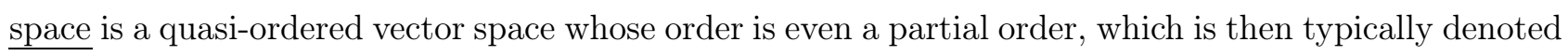
by $\leq$ instead of $\lesssim$. For every quasi-ordered vector space $V$, the convex cone (non-empty subset of a real vector space closed under addition and scalar multiplication with non-negative reals) of positive elements is $V^{+}:=\{v \in V \mid v \gtrsim 0\}$, and one can check that this describes a one-to-one correspondence between convex cones in $V$ and orders on $V$ that turn $V$ into a quasi-ordered vector space. From this point of view, $V$ is even an ordered vector space if and only if $V^{+} \cap\left(-V^{+}\right)=\{0\}$. A quasi-ordered vector space $V$ is called Archimedean if it has the following property: Whenever $v \lesssim \epsilon w$ holds for fixed $v \in V$ and $w \in V$ and all $\epsilon \in] 0, \infty[$, then $v \lesssim 0$.

The real vector space $\mathfrak{L}(V, W)$ of all linear maps $\Psi: V \rightarrow W$ between two quasi-ordered vector spaces is again a quasi-ordered vector space by declaring the positive elements to be precisely the increasing linear maps. Because of this, the increasing linear maps are called positive. Note that a linear map $\Psi: V \rightarrow W$ is increasing if and only if $\Psi(v) \in W^{+}$for all $v \in V^{+}$.

In ordered vector spaces it makes sense to discuss suprema and infima of arbitrary non-empty subsets. A Riesz space (or vector lattice) is an ordered vector space $\mathcal{R}$ in which suprema and infima of all pairs of elements exist. It is well-known that this is already the case if $\sup \{r,-r\}$ exists for all $r \in \mathcal{R}$. The usual notations $\vee$ and $\wedge$ for suprema and infima in Riesz spaces will be avoided and will be reserved for a similar concept that is introduced later for ordered *-algebras. Endowing Riesz spaces with an additional algebraic structure leads to e.g. the concept of $\Phi$-algebras, which are Archimedean Riesz spaces $\mathcal{R}$ endowed with a multiplication that turns $\mathcal{R}$ into a real unital associative algebra such that $r s \in \mathcal{R}^{+}$for all $r, s \in \mathcal{R}^{+}$and $\inf \{r t, s\}=\inf \{t r, s\}=0$ for all $r, s, t \in \mathcal{R}^{+}$with $\inf \{r, s\}=0$. 
Note that this property, applied twice with $t=r$ and $t=s$, especially implies that $r s=\inf \{r s, r s\}=0$ for all $r, s \in \mathcal{R}^{+}$with $\inf \{r, s\}=0$, and from $0=\inf \{\sup \{r,-r\}-r, \sup \{r,-r\}+r\}$ for all $r \in \mathcal{R}$ it follows that $(\sup \{r,-r\})^{2}=r^{2}$. One remarkable result about $\Phi$-algebras is a representation theorem as algebras of extended real-valued functions on compact Hausdorff spaces [5], and especially that $\Phi$-algebras are automatically commutative. This shows that $\Phi$-algebras are a good abstraction of lattice-ordered algebras of real-valued functions. There are also many similar notions of Riesz spaces with multiplication that have been studied extensively, most notably (almost) $f$-algebras. One essential takeaway is that a multiplication on an Archimedean Riesz space is automatically commutative under very mild assumptions of compatibility with the order $[1,3,6,9]$. This unfortunately means that such algebras are not suitable for the description of reasonably well-behaved non-commutative algebras of operators, which are the usual non-commutative analog of algebras of functions.

A ${ }^{*}$-vector space is a complex vector space $V$ endowed with an antilinear involution $\cdot{ }^{*}: V \rightarrow V$. An element $v$ of a ${ }^{*}$-vector space $V$ is called Hermitian if $v=v^{*}$ and the real linear subspace of Hermitian elements in $V$ is denoted by $V_{\mathrm{H}}$. Then $V=V_{\mathrm{H}} \oplus \mathrm{i} V_{\mathrm{H}}$ as a real vector space, and this decomposition can explicitly be described as $v=\operatorname{Re}(v)+\mathrm{i} \operatorname{Im}(v)$ with $\operatorname{Re}(v)=\frac{1}{2}\left(v+v^{*}\right)$ and $\operatorname{Im}(v)=\frac{1}{2 \mathrm{i}}\left(v-v^{*}\right)$ for all $v \in V$. The most obvious example of a ${ }^{*}$-vector space is of course given by $\mathbb{C}$ with complex conjugation - as *-involution. The complex vector space $\mathfrak{L}(V, W)$ of all linear maps $\Psi: V \rightarrow W$ between two ${ }^{*}$-vector spaces is again endowed with an antilinear involution defined by $\Psi^{*}(v):=\Psi\left(v^{*}\right)^{*}$ for all $\Psi \in \mathfrak{L}(V, W)$ and all $v \in V$. A linear map $\Psi: V \rightarrow W$ thus is Hermitian if and only if $\Psi\left(v^{*}\right)=\Psi(v)^{*}$ holds for all $v \in V$, or equivalently, if and only if $\Psi(v) \in W_{\mathrm{H}}$ for all $v \in V_{\mathrm{H}}$.

A ${ }^{*}$-algebra is a unital associative complex algebra $\mathcal{A}$ which is also a ${ }^{*}$-vector space such that $(a b)^{*}=b^{*} a^{*}$ holds for all $a, b \in \mathcal{A}$. Its unit is denoted by $\mathbb{1}$ or, more explicitly, by $\mathbb{1}_{\mathcal{A}}$, and is automatically Hermitian. Moreover, a unital *-homomorphism between two *-algebras is a unital homomorphism of algebras which is additionally Hermitian, and a unital *-subalgebra of a *-algebra is a unital subalgebra that is stable under .*. It is not explicitly required that $0 \neq \mathbb{1}$, but the only case in which this is not fulfilled is the not very interesting algebra $\{0\}$. For a subset $S \subseteq \mathcal{A}$ of a ${ }^{*}$-algebra $\mathcal{A}$, the commutant $S^{\prime}:=\left\{a \in \mathcal{A} \mid \forall_{s \in S}: s a=a s\right\}$ is a unital subalgebra, and even a unital *-subalgebra if $S$ is stable under **. If $S$ is commutative, then the bicommutant $S^{\prime \prime}$ is again commutative and $S \subseteq S^{\prime \prime} \subseteq S^{\prime}$. For example, the multiplicative inverse $a^{-1}$ of an invertible $a \in \mathcal{A}$ is in

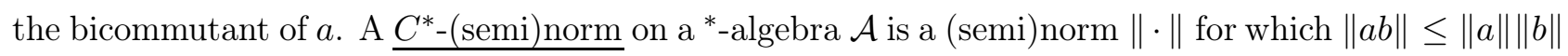

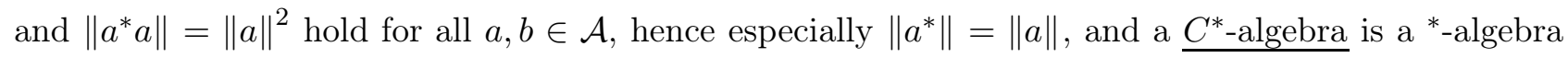
that is complete with respect to the topology of a $C^{*}$-norm.

A (quasi-)ordered ${ }^{*}$-vector space is a ${ }^{*}$-vector space $V$ whose real linear subspace of Hermitian elements $V_{\mathrm{H}}$ is endowed with an order that turns it into a (quasi-)ordered vector space. The properties of ordered vector spaces and linear functions between them, like being Archimedean or positive, apply to ordered *-vector spaces in the obvious way, i.e. they refer to the order on the Hermitian elements.

\section{Archimedean Ordered *-Algebras}

(Quasi-)ordered *-algebras are defined analogously to (quasi-)ordered *-vector spaces, and have already been studied in e.g. $[8,11]$ as "*-algebras that are equipped with an admissible wedge" in the context of 
*-algebras of unbounded operators and, especially in the commutative case and up to complexification, as "rings equipped with a quadratic module" in real algebraic geometry, see e.g. [10] for a survey. However, it is important to point out that with respect to quadratic modules, the term "Archimedean" unfortunately is used in a different way than with respect to ordered vector spaces.

Definition 3.1 A quasi-ordered ${ }^{*}$-algebra is $a^{*}$-algebra $\mathcal{A}$ whose real linear subspace $\mathcal{A}_{\mathrm{H}}$ is endowed with a quasi-order $\lesssim$ such that

$$
a+c \lesssim b+c, \quad d^{*} a d \lesssim d^{*} b d \quad \text { and } \quad 0 \lesssim \mathbb{1}
$$

hold for all $a, b, c \in \mathcal{A}_{\mathrm{H}}$ with $a \lesssim b$ and all $d \in \mathcal{A}$. An ordered $^{*}$-algebra is a quasi-ordered ${ }^{*}$-algebra $\mathcal{A}$ for which $\mathcal{A}_{\mathrm{H}}$ is partially ordered.

As *-algebras are required to have a unit, these axioms especially imply that every (quasi-)ordered *-algebra is a (quasi-)ordered *-vector space. Thus, a quasi-ordered *-algebra $\mathcal{A}$ will be called Archimedean if $\mathcal{A}_{\mathrm{H}}$ is Archimedean as a quasi-ordered vector space and we will especially be interested in positive Hermitian linear maps and positive unital ${ }^{*}$-homomorphisms between quasi-ordered ${ }^{*}$-algebras. Note that the set $\mathcal{A}_{\mathrm{H}}^{+}$of positive Hermitian elements of $\mathcal{A}$ generates $\mathcal{A}_{\mathrm{H}}$ as a real vector space because $4 a=(a+\mathbb{1})^{2}-(a-\mathbb{1})^{2}$ holds for all $a \in \mathcal{A}_{\mathrm{H}}$ and because $(a \pm \mathbb{1})^{2} \in \mathcal{A}_{\mathrm{H}}^{+}$. Moreover, one easily checks that

$$
\lambda a+\mu b \in \mathcal{A}_{\mathrm{H}}^{+}, \quad d^{*} a d \in \mathcal{A}_{\mathrm{H}}^{+} \quad \text { and } \quad \mathbb{1} \in \mathcal{A}_{\mathrm{H}}^{+}
$$

hold for all $a, b \in \mathcal{A}_{\mathrm{H}}^{+}, d \in \mathcal{A}$ and scalars $\lambda, \mu \in\left[0, \infty\left[\right.\right.$. Conversely, if $\mathcal{A}$ is a ${ }^{*}$-algebra and $\mathcal{A}_{\mathrm{H}}^{+}$an arbitrary subset of $\mathcal{A}_{\mathrm{H}}$ that fulfils these three conditions (3.2), then there is a unique order on $\mathcal{A}_{\mathrm{H}}$ such that $\mathcal{A}$ becomes a quasi-ordered ${ }^{*}$-algebra whose set of positive Hermitian elements is precisely this set $\mathcal{A}_{\mathrm{H}}^{+}$. This order is given for $a, b \in \mathcal{A}_{\mathrm{H}}$ by $a \lesssim b$ iff $b-a \in \mathcal{A}_{\mathrm{H}}^{+}$.

Again, the most obvious example of an ordered ${ }^{*}$-algebra is $\mathbb{C}$ with the usual order on $\mathbb{C}_{\mathrm{H}} \cong \mathbb{R}$. More interesting ones are:

Example 3.2 Let $X$ be a non-empty set and $\mathbb{C}^{X}$ the unital *-algebra of all complex-valued functions on $X$ with the pointwise operations. Then $\mathbb{C}^{X}$ with the pointwise order on its Hermitian elements, i.e. $f \leq g$ if and only if $f(x) \leq g(x)$ for all $x \in X$, is an Archimedean ordered *-algebra. Consequently, all unital *-subalgebras of $\mathbb{C}^{X}$ with this pointwise order are Archimedean ordered *-algebras as well.

Special cases of such ordered ${ }^{*}$-algebras of functions are of course those of continuous functions, denoted by $\mathscr{C}(X)$ if $X$ is a topological space. Another special case are polynomials, which demonstrate that there can be, in general, many possible orders on the same *-algebra:

Example 3.3 Let $\mathbb{C}\left[x_{1}, \ldots, x_{N}\right]$ with $N \in \mathbb{N}$ be the ${ }^{*}$-algebra of complex polynomials in $N$ Hermitian variables $x_{1}, \ldots, x_{N}$, i.e. the ${ }^{*}$-involution is given by complex conjugation of all coefficients. For every

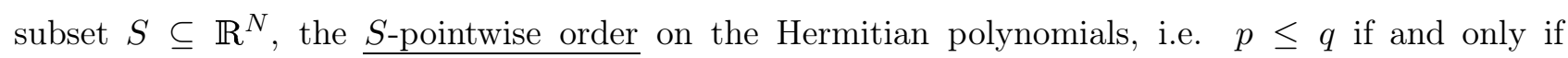
$p\left(s_{1}, \ldots, s_{N}\right) \leq q\left(s_{1}, \ldots, s_{N}\right)$ for all $\left(s_{1}, \ldots, s_{N}\right) \in S$, turns $\mathbb{C}\left[x_{1}, \ldots, x_{N}\right]$ into an Archimedean quasiordered *-algebra, which is even an Archimedean ordered *-algebra e.g. if $S$ has non-empty interior.

Non-commutative examples are provided by ${ }^{*}$-algebras of operators, i.e. $O^{*}$-algebras: 
Example 3.4 Let $\mathcal{D}$ be a complex pre-Hilbert space with inner product $\langle\cdot \mid \cdot\rangle$ (antilinear in the first, linear in the second argument), then a linear endomorphism $a: \mathcal{D} \rightarrow \mathcal{D}$ is said to be adjointable if there exists a (necessarily unique) linear map $a^{*}: \mathcal{D} \rightarrow \mathcal{D}$ such that $\left\langle a^{*}(\xi) \mid \eta\right\rangle=\langle\xi \mid a(\eta)\rangle$ holds for all $\xi, \eta \in \mathcal{D}$. In this case, $a^{*}$ is called the adjoint endomorphism. The set of all adjointable linear endomorphisms on $\mathcal{D}$ is a *-algebra and becomes an Archimedean ordered *-algebra, denoted $\mathfrak{L}^{*}(\mathcal{D})$, with the usual order of Hermitian operators on $\mathcal{D}$, i.e. $a \leq b$ if and only if $\langle\xi \mid a(\xi)\rangle \leq\langle\xi \mid b(\xi)\rangle$ for all $\xi \in \mathcal{D}$. Consequently, all unital ${ }^{*}$-subalgebras of $\mathfrak{L}^{*}(\mathcal{D})$ are Archimedean ordered *-algebras as well. These are the $O^{*}$-algebras on $\mathcal{D}$, see e.g. the monograph [11] for more details.

In these first examples, the order is essentially determined by positive Hermitian linear functionals, namely the evaluation functionals at points of $X$ and $S$ in Examples 3.2 and 3.3, or the vector functionals $\mathfrak{L}^{*}(\mathcal{D}) \ni a \mapsto\langle\xi \mid a(\xi)\rangle \in \mathbb{C}$ in Example 3.4. But there are also other types of examples:

Example 3.5 Let $\mathcal{A}$ be a unital *-algebra, $G \subseteq \mathcal{A}_{\mathrm{H}}$ and define $\left\langle\langle G\rangle_{\mathrm{pos}}\right.$ as

$$
\langle G\rangle_{\mathrm{pos}}:=\left\{\sum_{n=1}^{N} a_{n}^{*} g_{n} a_{n} \mid N \in \mathbb{N} ; g_{1}, \ldots, g_{N} \in G \cup\{\mathbb{1}\}, a_{1}, \ldots, a_{N} \in \mathcal{A}\right\} .
$$

Then setting $\mathcal{A}_{\mathrm{H}}^{+}:=\left\langle\langle G\rangle_{\text {pos }}\right.$ turns $\mathcal{A}$ into a quasi-ordered ${ }^{*}$-algebra. This order on $\mathcal{A}$ will be called the order generated by $G$, and $\left\langle\langle G\rangle_{\text {pos }}\right.$ is the smallest (with respect to inclusion) choice of positive Hermitian elements that contains $G$ and with which $\mathcal{A}$ becomes a quasi-ordered *-algebra. Especially for $G=\emptyset$ we write

$$
\mathcal{A}_{\mathrm{H}}^{++}:=\langle\emptyset\rangle_{\mathrm{pos}}=\left\{\sum_{n=1}^{N} a_{n}^{*} a_{n} \mid N \in \mathbb{N} ; a_{1}, \ldots, a_{N} \in \mathcal{A}\right\}
$$

and call the elements of $\mathcal{A}_{\mathrm{H}}^{++}$algebraically positive.

There are many strong results (called "Positivstellensätze") in real algebraic geometry that link orders on ${ }^{*}$-algebras that are induced by positive Hermitian linear functionals like in Examples 3.2, 3.3 and 3.4 to orders generated by a set of positive elements like in Example 3.5. These include the classical Positivstellensatz of Krivine and Stengle for polynomials but also newer results for non-commutative *algebras like $[13,14]$.

Choosing the order on a ${ }^{*}$-algebra $\mathcal{A}$ for which $\mathcal{A}_{\mathrm{H}}^{+}=\mathcal{A}_{\mathrm{H}}^{++}$yields a canonical way to construct a quasi-ordered *-algebra out of any ${ }^{*}$-algebra. For example, the canonical order on $C^{*}$-algebras can be described like this. There is also another canonical (yet pathological) choice, namely $\mathcal{A}_{\mathrm{H}}^{+}=\mathcal{A}_{\mathrm{H}}$. For a general quasi-ordered *-algebra $\mathcal{A}$ one clearly has $\mathcal{A}_{\mathrm{H}}^{++} \subseteq \mathcal{A}_{\mathrm{H}}^{+} \subseteq \mathcal{A}_{\mathrm{H}}$, but it is well-known that these extreme cases may coincide:

Example 3.6 Let $\mathbb{S}:=\{z \in \mathbb{C}|| z \mid=1\}$ and let $\mathcal{A}$ be the unital associative algebra $\mathcal{A}:=\mathscr{C}(\mathbb{S})$, but endowed with the ${ }^{*}$-involution $f^{*}:=-\circ f \circ \tau$ for all $f \in \mathscr{C}(\mathbb{S})$ (instead of the usual pointwise one $\left.f^{*}:=-\circ f\right)$, where $\tau: \mathbb{S} \rightarrow \mathbb{S}$ is $z \mapsto \tau(z):=-z$. This way $\mathscr{C}(\mathbb{S})$ indeed becomes a ${ }^{*}$-algebra. The usual norm $\|f\|_{\max }:=\max _{z \in \mathbb{S}}|f(z)|$ turns $\mathscr{C}(\mathbb{S})$ into a Banach space and makes multiplication and ${ }^{*}$-involution continuous. However, $\mathrm{id}_{\mathbb{S}}$ describes a function in $\mathscr{C}(\mathbb{S})$ for which $-\mathbb{1}_{\mathbb{S}}=\left(\mathrm{id}_{\mathbb{S}}\right)^{*} \mathrm{id}_{\mathbb{S}} \in \mathcal{A}_{\mathrm{H}}^{++}$ holds, thus $\mathcal{A}_{\mathrm{H}}=\mathcal{A}_{\mathrm{H}}^{++}-\mathcal{A}_{\mathrm{H}}^{++}=\mathcal{A}_{\mathrm{H}}^{++}$. 
Finally, there is a standard example of a non-Archimedean ordered *-algebra:

Example 3.7 The commutative unital subalgebra

$$
\mathcal{A}:=\left\{M_{a, b}:=\left(\begin{array}{ll}
a & b \\
0 & a
\end{array}\right) \in \mathbb{C}^{2 \times 2} \mid a, b \in \mathbb{C}\right\}
$$

of the matrix algebra $\mathbb{C}^{2 \times 2}$ with elementwise complex conjugation as ${ }^{*}$-involution becomes a ${ }^{*}$-algebra. Its algebraically positive elements are

$$
\mathcal{A}_{\mathrm{H}}^{++}=\left\{M_{a, b} \mid a, b \in \mathbb{R} \text { with } a>0 \text { or } a=b=0\right\}
$$

and $\mathcal{A}$ with the algebraic order is an ordered *-algebra, but not Archimedean because $M_{0,1} \leq \epsilon M_{1,0}$ for all $\epsilon \in] 0, \infty\left[\right.$. Note especially that $M_{0,1}$ is a non-zero Hermitian element that squares to 0 .

Examples 3.6 and 3.7 already indicate that one should not expect to be able to prove many strong results for quasi-ordered *-algebras without any additional assumptions. Nevertheless, there is at least a possibility to characterize the pathological elements and in many cases one can eventually get rid of them by taking a suitable quotient. This way it will become clear that an order on a ${ }^{*}$-algebra can be seen as a generalization of a $C^{*}$-norm. This follows essentially [4], but caution is advised because of the different usage of the term "Archimedean" there:

Lemma 3.8 Let $\mathcal{A}$ be a quasi-ordered ${ }^{*}$-algebra, $a \in \mathcal{A}_{\mathrm{H}}$ and $\left.\lambda \in\right] 0, \infty\left[\right.$, then $a^{2} \lesssim \lambda^{2} \mathbb{1}$ if and only if $-\lambda \mathbb{1} \lesssim a \lesssim \lambda \mathbb{1}$. If $\mathcal{A}$ is Archimedean, then this equivalence also holds for $\lambda=0$.

Proof: For $\lambda \in] 0, \infty[$ this is essentially [4, Lemma 3.1], an immediate consequence of the identities

$$
\lambda \mathbb{1} \pm a=\frac{\lambda^{2} \mathbb{1}-a^{2}+(\lambda \mathbb{1} \pm a)^{2}}{2 \lambda}
$$

and

$$
\lambda^{2} \mathbb{1}-a^{2}=\frac{(\lambda \mathbb{1}+a)(\lambda \mathbb{1}-a)(\lambda \mathbb{1}+a)+(\lambda \mathbb{1}-a)(\lambda \mathbb{1}+a)(\lambda \mathbb{1}-a)}{2 \lambda} .
$$

So $a^{2} \lesssim 0$ implies $-\epsilon \mathbb{1} \lesssim a \lesssim \epsilon \mathbb{1}$ and $0 \lesssim a \lesssim 0$ implies $a^{2} \lesssim \epsilon^{2} \mathbb{1}$ for all $\left.\epsilon \in\right] 0, \infty[$. If $\mathcal{A}$ is Archimedean, then this shows that $a^{2} \lesssim 0$ and $0 \lesssim a \lesssim 0$ are also equivalent.

Proposition 3.9 Let $\mathcal{A}$ be an Archimedean ordered ${ }^{*}$-algebra and $a \in \mathcal{A}_{\mathrm{H}}$ nilpotent, then $a=0$.

Proof: Let $n \in \mathbb{N}$ be the minimal exponent for which $a^{n}=0$. Then $n$ is odd because otherwise $0 \leq a^{n / 2} \leq 0$ by the previous Lemma 3.8, which contradicts minimality of $n$. But $a^{n+1}=0$ now implies $0 \leq a^{(n+1) / 2} \leq 0$, so $n=1$ by minimality of $n$.

In Example 3.7 we have seen that nilpotent Hermitian elements can indeed occur in non-Archimedean ordered *-algebras. Like in [4] we define:

Definition 3.10 Let $\mathcal{A}$ be a quasi-ordered ${ }^{*}$-algebra, then define the map $\|\cdot\|_{\infty}: \mathcal{A} \rightarrow[0, \infty]$,

$$
a \mapsto\|a\|_{\infty}:=\inf \{\lambda \in] 0, \infty\left[\mid a^{*} a \lesssim \lambda^{2} \mathbb{1}\right\}
$$


where it is understood that the infimum of the empty set is $\infty$. An element $a \in \mathcal{A}$ is called uniformly

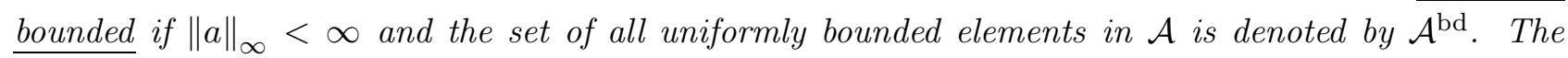

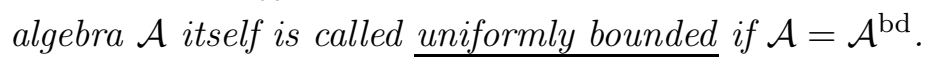

Lemma 3.8 immediately gives an alternative description of $\|\cdot\|_{\infty}$ on Hermitian elements:

Proposition 3.11 Let $\mathcal{A}$ be a quasi-ordered ${ }^{*}$-algebra and $a \in \mathcal{A}_{\mathrm{H}}$, then

$$
\|a\|_{\infty}=\inf \{\lambda \in] 0, \infty[\mid-\lambda \mathbb{1} \lesssim a \lesssim \lambda \mathbb{1}\}
$$

where again the infimum of the empty set is $\infty$.

In the Archimedean case, these infima are even minima:

Proposition 3.12 Let $\mathcal{A}$ be an Archimedean quasi-ordered ${ }^{*}$-algebra and $a \in \mathcal{A}^{\text {bd }}$, then

$$
a^{*} a \lesssim\|a\|_{\infty}^{2} \mathbb{1}
$$

If even $a \in\left(\mathcal{A}^{\mathrm{bd}}\right)_{\mathrm{H}}$, then also

$$
-\|a\|_{\infty} \mathbb{1} \lesssim a \lesssim\|a\|_{\infty} \mathbb{1}
$$

Proof: From the definition of $\|a\|_{\infty}$ one sees that $a^{*} a \lesssim\left(\|a\|_{\infty}^{2}+\epsilon\right) \mathbb{1}$ for all $\left.\epsilon \in\right] 0, \infty\left[\right.$, hence $a^{*} a \lesssim$ $\|a\|_{\infty}^{2} \mathbb{1}$ as $\mathcal{A}$ is Archimedean. If $a$ is even Hermitian, then this implies $-\|a\|_{\infty} \mathbb{1} \lesssim a \lesssim\|a\|_{\infty} \mathbb{1}$ by Lemma 3.8 again.

The crucial property of $\|\cdot\|_{\infty}$ is that it yields a $C^{*}$-(semi)norm on the uniformly bounded elements. Recall that a ${ }^{*}$-ideal of a ${ }^{*}$-algebra $\mathcal{A}$ is a linear subspace $\mathcal{I} \subseteq \mathcal{A}$ that is stable under the ${ }^{*}$-involution and fulfils $b a \in \mathcal{I}$ for all $a \in \mathcal{A}$ and all $b \in \mathcal{I}$ (thus also $a b=\left(b^{*} a^{*}\right)^{*} \in \mathcal{I}$ for all $a \in \mathcal{A}$ and all $b \in \mathcal{I}$ ).

Proposition 3.13 Let $\mathcal{A}$ be a quasi-ordered ${ }^{*}$-algebra, then $\mathcal{A}^{\text {bd }}$ is a unital ${ }^{*}$-subalgebra of $\mathcal{A}$, and the restriction of $\|\cdot\|_{\infty}$ to $\mathcal{A}^{\mathrm{bd}}$ is a $C^{*}$-seminorm. Its kernel $\mathcal{K}:=\left\{a \in \mathcal{A}\|\| a \|_{\infty}=0\right\}$ is $a{ }^{*}$-ideal of $\mathcal{A}^{\mathrm{bd}}$. If $\mathcal{A}$ is Archimedean, then $\mathcal{K}$ is even $a^{*}$-ideal of whole $\mathcal{A}$, and if $\mathcal{A}$ is an Archimedean ordered ${ }^{*}$-algebra, then $\mathcal{K}=\{0\}$ so that $\|\cdot\|_{\infty}$ is a $C^{*}$-norm on $\mathcal{A}^{\mathrm{bd}}$.

Proof: The claims for general, not necessarily Archimedean $\mathcal{A}$ have been proven in [4, Thm. 3.2]. For convenience of the reader, the details are given here as well:

From the definition of $\|\cdot\|_{\infty}$ it is clear that $\mathbb{1} \in \mathcal{A}^{\text {bd }}$ with $\|\mathbb{1}\|_{\infty} \leq 1$ and that $\alpha a \in \mathcal{A}^{\text {bd }}$ with $\|\alpha a\|_{\infty}=|\alpha|\|a\|_{\infty}$ for all $a \in \mathcal{A}^{\text {bd }}$ and all $\alpha \in \mathbb{C} \backslash\{0\}$, as well as for $\alpha=0$ because clearly $\|0\|_{\infty}=0$. Now given $a, b \in \mathcal{A}^{\text {bd }}$ and $\left.\lambda, \mu \in\right] 0, \infty\left[\right.$ such that $a^{*} a \lesssim \lambda^{2} \mathbb{1}$ and $b^{*} b \lesssim \mu^{2} \mathbb{1}$, then

$$
\begin{aligned}
&(\lambda+\mu)^{2} \mathbb{1}-(a+b)^{*}(a+b)= \\
&=\left(1+\frac{\mu}{\lambda}\right)\left(\lambda^{2} \mathbb{1}-a^{*} a\right)+\left(1+\frac{\lambda}{\mu}\right)\left(\mu^{2} \mathbb{1}-b^{*} b\right)+\left(\frac{\sqrt{\lambda}}{\sqrt{\mu}} b-\frac{\sqrt{\mu}}{\sqrt{\lambda}} a\right)^{*}\left(\frac{\sqrt{\lambda}}{\sqrt{\mu}} b-\frac{\sqrt{\mu}}{\sqrt{\lambda}} a\right)
\end{aligned}
$$

is positive, so $\|a+b\|_{\infty} \leq \lambda+\mu$. Moreover, $(a b)^{*}(a b)=b^{*} a^{*} a b \lesssim \lambda^{2} b^{*} b \lesssim(\lambda \mu)^{2} \mathbb{1}$ shows that $\|a b\|_{\infty} \leq \lambda \mu$. Thus $\|a+b\|_{\infty} \leq\|a\|_{\infty}+\|b\|_{\infty}$ and $\|a b\|_{\infty} \leq\|a\|_{\infty}\|b\|_{\infty}$, and especially $a+b, a b \in \mathcal{A}^{\mathrm{bd}}$. So $\mathcal{A}^{\text {bd }}$ is a unital subalgebra of $\mathcal{A}$ and $\|\cdot\|_{\infty}$ a submultiplicative seminorm on $\mathcal{A}^{\text {bd }}$. 
In order to show that $\mathcal{A}^{\text {bd }}$ is stable under $*^{*}$, let $a \in \mathcal{A}^{\text {bd }}$ be given as well as $\left.\lambda \in\right] 0, \infty[$ such that $a^{*} a \lesssim \lambda^{2} \mathbb{1}$. Then

$$
0 \lesssim \frac{\left(\lambda^{2} \mathbb{1}-a a^{*}\right)^{2}}{\lambda^{2}}=\lambda^{2} \mathbb{1}-2 a a^{*}+a \frac{a^{*} a}{\lambda^{2}} a^{*} \lesssim \lambda^{2} \mathbb{1}-a a^{*}
$$

shows that $\left\|a^{*}\right\|_{\infty} \leq \lambda$. It follows that $\mathcal{A}^{\text {bd }}$ is a unital ${ }^{*}$-subalgebra of $\mathcal{A}$ and $\left\|a^{*}\right\|_{\infty}=\|a\|_{\infty}$ for all $a \in \mathcal{A}$ because $\cdot{ }^{*}$ is an involution.

The $C^{*}$-property also is fulfilled: The increasing and continuous map ${ }^{2}:[0, \infty[\rightarrow[0, \infty[$ commutes with infima so that Definition 3.10 yields $\|a\|_{\infty}^{2}=\inf \left\{\lambda^{2} \in\right] 0, \infty\left[\mid a^{*} a \lesssim \lambda^{2} \mathbb{1}\right\}$ for all $a \in \mathcal{A}^{\text {bd }}$, which coincides with $\left\|a^{*} a\right\|_{\infty}$ by Proposition 3.11. So $\|\cdot\|_{\infty}$ is indeed a $C^{*}$-seminorm on $\mathcal{A}^{\text {bd }}$ and its kernel $\mathcal{K}$ is a ${ }^{*}$-ideal of $\mathcal{A}^{\text {bd }}$.

Now given $a, b \in \mathcal{A}$ such that $\|a\|_{\infty}=0$, then $(a b)^{*}(a b)=b^{*} a^{*} a b \lesssim \epsilon^{2} b^{*} b$ holds for all $\left.\epsilon \in\right] 0, \infty[$, which implies $(a b)^{*}(a b) \lesssim 0$ if $\mathcal{A}$ is additionally Archimedean. In this case $\|a b\|_{\infty}=0$ so that $\mathcal{K}$ is even a ${ }^{*}$-ideal of $\mathcal{A}$.

Finally, assume that $\mathcal{A}$ is even an Archimedean ordered ${ }^{*}$-algebra and let $a \in \mathcal{K}$ be given. If $a$ is Hermitian, then $a=0$ by Proposition 3.12. Otherwise $a$ can be expressed as the linear combination $a=\operatorname{Re}(a)+\mathrm{i} \operatorname{Im}(a)$ of Hermitian elements $\operatorname{Re}(a), \operatorname{Im}(a) \in \mathcal{K}$, which are both 0 so that again $a=0$.

So we see that uniformly bounded Archimedean ordered ${ }^{*}$-algebras with the norm $\|\cdot\|_{\infty}$ are pre- $C^{*}$-algebras (i.e. * ${ }^{*}$-algebras endowed with a $C^{*}$-norm). Using some standard results about $C^{*}$-algebras, e.g. the possibility to represent every $C^{*}$-algebra as a ${ }^{*}$-algebra of bounded operators on a Hilbert space by the Gelfand-Naimark theorem, one can also show that the converse is true as well: every pre- $C^{*}$-algebra with the canonical order inherited from its completion to a $C^{*}$-algebra is a uniformly bounded Archimedean ordered *-algebra. It will be interesting to extend the concept of completeness of a $C^{*}$-algebra to general Archimedean ordered ${ }^{*}$-algebras. While $\|\cdot\|_{\infty}$ is finite only on the uniformly bounded elements, and thus does not describe a norm on all Archimedean ordered ${ }^{*}$-algebras, it still allows to construct a translation-invariant metric:

Definition 3.14 Let $\mathcal{A}$ be an Archimedean ordered ${ }^{*}$-algebra, then the uniform metric on $\mathcal{A}$ is defined as the $\operatorname{map} \mathrm{d}_{\infty}: \mathcal{A} \times \mathcal{A} \rightarrow[0, \infty[$,

$$
(a, b) \mapsto \mathrm{d}_{\infty}(a, b):=\min \left\{\|a-b\|_{\infty}, 1\right\}
$$

All metric notions will always refer to this uniform metric, and $\mathcal{A}$ is especially called uniformly complete if it is complete with respect to $\mathrm{d}_{\infty}$.

Note that it is easy to check that $\mathrm{d}_{\infty}$ is indeed a translation-invariant metric.

In this language, $C^{*}$-algebras are the uniformly bounded and uniformly complete Archimedean ordered *-algebras. However, neither the product, nor the left or right multiplication with a fixed element are continuous in the general case: Consider the ${ }^{*}$-algebra $\mathbb{C}[x]$ of polynomials in one Hermitian element $x$ like in Example 3.3 with the $\mathbb{R}$-pointwise ordering. Then $\lim _{n \rightarrow \infty} \mathbb{1} / n=0$ but the sequence $\mathbb{N} \ni n \mapsto x / n \in \mathbb{C}[x]_{\mathrm{H}}$ does not converge. Nevertheless, this metric is still sufficiently well-behaved for some purposes. For example, it is easy to see that every positive unital *-homomorphism between Archimedean ordered *-algebras is automatically continuous with respect to the uniform metric, because this metric is induced by the order. Moreover: 
Lemma 3.15 Let $\mathcal{A}$ be a quasi-ordered ${ }^{*}$-algebra and $a, b \in \mathcal{A}$, then

$$
a^{*} b+b^{*} a \lesssim \chi^{-2} a^{*} a+\chi^{2} b^{*} b
$$

holds for all $\chi \in] 0, \infty[$.

Proof: This follows from $0 \lesssim\left(\chi^{-1} a-\chi b\right)^{*}\left(\chi^{-1} a-\chi b\right)=\chi^{-2} a^{*} a-a^{*} b-b^{*} a+\chi^{2} b^{*} b$.

Proposition 3.16 Let $\mathcal{A}$ be an Archimedean ordered ${ }^{*}$-algebra and $S \subseteq \mathcal{A}_{\mathrm{H}}$, then the space $\mathcal{A}_{\mathrm{H}}$ of Hermitian elements, the space $\mathcal{A}^{\mathrm{bd}}$ of uniformly bounded elements, the commutant $S^{\prime}$, the bicommutant $S^{\prime \prime}$ and the sets $\left\{a \in \mathcal{A}_{\mathrm{H}} \mid a \leq s\right.$ for all $\left.s \in S\right\}$ and $\left\{a \in \mathcal{A}_{\mathrm{H}} \mid a \geq s\right.$ for all $\left.s \in S\right\}$ are closed in $\mathcal{A}$ with respect to the uniform metric.

Proof: Using that $\|\operatorname{Re}(a)\|_{\infty} \leq\|a\|_{\infty}$ and $\|\operatorname{Im}(a)\|_{\infty} \leq\|a\|_{\infty}$ hold for all $a \in \mathcal{A}^{\text {bd }}$, it is easy to check that the $\mathbb{R}$-linear projectors $\operatorname{Re}, \operatorname{Im}: \mathcal{A} \rightarrow \mathcal{A}$ are continuous and thus $\mathcal{A}_{\mathrm{H}}=\operatorname{Im}^{-1}(\{0\})$ is closed.

Now consider a sequence $\left(a_{n}\right)_{n \in \mathbb{N}}$ in $\mathcal{A}_{\mathrm{H}}$ that converges against some $\hat{a}:=\lim _{n \rightarrow \infty} a_{n} \in \mathcal{A}_{\mathrm{H}}$ and let $\epsilon \in] 0, \infty\left[\right.$ be given, then there exists an $n \in \mathbb{N}$ such that $\left\|\hat{a}-a_{n}\right\|_{\infty} \leq \epsilon$, i.e. $-\epsilon \mathbb{1} \leq \hat{a}-a_{n} \leq \epsilon \mathbb{1}$ by Proposition 3.12. If all $a_{n}$ with $n \in \mathbb{N}$ are uniformly bounded, then this shows that $\hat{a}$ is also uniformly bounded, so $\mathcal{A}^{\text {bd }} \cap \mathcal{A}_{\mathrm{H}}$ and $\mathcal{A}^{\text {bd }}=\operatorname{Re}^{-1}\left(\mathcal{A}^{\text {bd }} \cap \mathcal{A}_{\mathrm{H}}\right) \cap \operatorname{Im}^{-1}\left(\mathcal{A}^{\text {bd }} \cap \mathcal{A}_{\mathrm{H}}\right)$ are closed in $\mathcal{A}$.

Moreover, let $s \in \mathcal{A}_{\mathrm{H}}$ be given. If $a_{n} \leq s$ for $n \in \mathbb{N}$, then $\hat{a} \leq a_{n}+\epsilon \mathbb{1} \leq s+\epsilon \mathbb{1}$, and if $a_{n} \geq s$ for $n \in \mathbb{N}$, then $\hat{a} \geq a_{n}-\epsilon \mathbb{1} \geq s-\epsilon \mathbb{1}$. If $a_{n} \in\{s\}^{\prime}$ for $n \in \mathbb{N}$, then

$$
\mathrm{i}(\hat{a} s-s \hat{a})=\left(\hat{a}-a_{n}\right)(\mathrm{i} s)+(-\mathrm{i} s)\left(\hat{a}-a_{n}\right) \leq \epsilon^{-1}\left(\hat{a}-a_{n}\right)^{2}+\epsilon s^{2} \leq \epsilon\left(\mathbb{1}+s^{2}\right)
$$

by Lemma 3.15 with $\chi=\sqrt{\epsilon}$ and Lemma 3.8. As $\{-s\}^{\prime}=\{s\}^{\prime}$, the same estimate holds with $-s$ in place of $s$, so $-\epsilon\left(\mathbb{1}+s^{2}\right) \leq \mathrm{i}(\hat{a} s-s \hat{a}) \leq \epsilon\left(\mathbb{1}+s^{2}\right)$.

Using that $\mathcal{A}$ is Archimedean, these estimates show that $\left\{a \in \mathcal{A}_{\mathrm{H}} \mid a \leq s\right\},\left\{a \in \mathcal{A}_{\mathrm{H}} \mid a \geq s\right\}$, and $\{s\}^{\prime} \cap \mathcal{A}_{\mathrm{H}}$ are closed in $\mathcal{A}$. As $\{s\}^{\prime}=\operatorname{Re}^{-1}\left(\{s\}^{\prime} \cap \mathcal{A}_{\mathrm{H}}\right) \cap \operatorname{Im}^{-1}\left(\{s\}^{\prime} \cap \mathcal{A}_{\mathrm{H}}\right)$ also $\{s\}^{\prime}$ is closed in $\mathcal{A}$. Consequently, intersections of such sets, and especially $\left\{a \in \mathcal{A}_{\mathrm{H}} \mid a \leq s\right.$ for all $\left.s \in S\right\}$, $\left\{a \in \mathcal{A}_{\mathrm{H}} \mid a \geq s\right.$ for all $\left.s \in S\right\}$ and $S^{\prime}$ are closed. From $S \subseteq \mathcal{A}_{\mathrm{H}}$ it follows that $S^{\prime}$ is stable under $\cdot^{*}$, and thus $S^{\prime \prime}=\left(S^{\prime} \cap \mathcal{A}_{\mathrm{H}}\right)^{\prime}$ is also closed in $\mathcal{A}$.

\section{$4 \quad$ Radical and Symmetric Ordered *-Algebras}

The only compatibility between order and multiplication that has been discussed so far is the axiom of quasi-ordered ${ }^{*}$-algebras $\mathcal{A}$ that $b^{*} a b \in \mathcal{A}_{\mathrm{H}}^{+}$for all $a \in \mathcal{A}_{\mathrm{H}}^{+}$and all $b \in \mathcal{A}$. If the order is sufficiently nice (especially antisymmetric and Archimedean), then this is indeed enough to guarantee that the elements of $\mathcal{A}$ essentially behave like adjointable endomorphisms on a pre-Hilbert space, which can be made rigorous by a representation theorem like in [15]. However, it is well-known that such *-algebras of (unbounded) adjointable endomorphisms can still exhibit some unexpected behaviour because Hermitian endomorphisms need not be essentially self-adjoint. Because of this, it will be necessary to introduce another compatibility between order and multiplication that gurantees that commuting elements essentially behave like complex-valued functions (see again [15]): 
Definition 4.1 Let $\mathcal{A}$ be an ordered ${ }^{*}$-algebra, then an element $a \in \mathcal{A}_{\mathrm{H}}$ is called coercive if there exists an $\epsilon \in] 0, \infty\left[\right.$ such that $a \geq \epsilon \mathbb{1}$. An ordered ${ }^{*}$-algebra $\mathcal{A}$ is called radical if the following is fulfilled: Whenever $a, b \in \mathcal{A}_{\mathrm{H}}$ are two commuting elements such that $a$ is coercive and $a b \geq 0$, then $b \geq 0$.

One obvious example of radical Archimedean ordered *-algebras are function algebras like in Example 3.2. Non-commutative examples will be constructed later on. Some basic observations about radical Archimedean ordered *-algebras are:

Proposition 4.2 Let $\mathcal{A}$ be a radical Archimedean ordered ${ }^{*}$-algebra, a, $b \in \mathcal{A}_{\mathrm{H}}$ commuting and $a \geq 0$. Then $b^{2} \leq a^{2}$ is equivalent to $-a \leq b \leq a$.

Proof: One argues like in the proof of Lemma 3.8: First assume that $b^{2} \leq a^{2}$, then also $b^{2} \leq(a+\epsilon \mathbb{1})^{2}$ for all $\epsilon \in] 0, \infty[$, so

$$
2(a+\epsilon \mathbb{1})(a+\epsilon \mathbb{1} \pm b)=(a+\epsilon \mathbb{1})^{2}-b^{2}+(a+\epsilon \mathbb{1} \pm b)^{2} \geq 0
$$

As $\mathcal{A}$ is radical, this shows that $a+\epsilon \mathbb{1} \pm b \geq 0$, and then $a \pm b \geq 0$ because $\mathcal{A}$ is also Archimedean; so $-a \leq b \leq a$. Conversely, if $-a \leq b \leq a$, then also $-(a+\epsilon \mathbb{1}) \leq b \leq a+\epsilon \mathbb{1}$ for all $\epsilon \in] 0,1]$, so

$$
2(a+\epsilon \mathbb{1})\left((a+\epsilon \mathbb{1})^{2}-b^{2}\right)=(a+\epsilon \mathbb{1}+b)(a+\epsilon \mathbb{1}-b)(a+\epsilon \mathbb{1}+b)+(a+\epsilon \mathbb{1}-b)(a+\epsilon \mathbb{1}+b)(a+\epsilon \mathbb{1}-b) \geq 0 .
$$

As $\mathcal{A}$ is radical, this shows that $(a+\epsilon \mathbb{1})^{2}-b^{2} \geq 0$, so $b^{2} \leq(a+\epsilon \mathbb{1})^{2} \leq a^{2}+\epsilon(2 a+\mathbb{1})$. It follows that $b^{2} \leq a^{2}$ because $\mathcal{A}$ is Archimedean.

Corollary 4.3 If $\mathcal{A}$ is a radical Archimedean ordered ${ }^{*}$-algebra and $a, b \in \mathcal{A}_{\mathrm{H}}^{+}$commute, then ab $\geq 0$.

Proof: From $-(a+b) \leq a-b \leq a+b$ we get $(a-b)^{2} \leq(a+b)^{2}$, so $4 a b=(a+b)^{2}-(a-b)^{2} \geq 0$

Note that even in the matrix *-algebra $\mathbb{C}^{2 \times 2}$ with the composition of complex conjugation and transposition as *-involution and the usual order on the Hermitian matrices, which is a $C^{*}$-algebra and certainly should be regarded as one of the most well-behaved ordered ${ }^{*}$-algebras, there exist Hermitian (but not commuting) matrices $a$ and $b$ with $0 \leq a \leq b$ which do not fulfil $a^{2} \leq b^{2}$. A standard example is

$$
a=\left(\begin{array}{ll}
2 & 2 \\
2 & 2
\end{array}\right) \quad \text { and } \quad b=\left(\begin{array}{ll}
6 & 0 \\
0 & 3
\end{array}\right) .
$$

Because of this, one should not expect an analog of Proposition 4.2 to be fulfilled for non-commutating Hermitian elements in well-behaved examples. Using results from real algebraic geometry, the above Corollary 4.3 can be improved significantly, which demonstrates the importance of the radical-property:

Proposition 4.4 Let $\mathcal{A}$ be a radical Archimedean ordered ${ }^{*}$-algebra, $a_{1}, \ldots, a_{N} \in \mathcal{A}_{\mathrm{H}}$ with $N \in \mathbb{N}$ pairwise commuting and $p_{1}, \ldots, p_{M} \in \mathbb{R}\left[x_{1}, \ldots, x_{N}\right] \cong \mathbb{C}\left[x_{1}, \ldots, x_{N}\right]_{\mathrm{H}}$ with $M \in \mathbb{N}$ polynomials fulfilling $p_{m}\left(a_{1}, \ldots, a_{N}\right) \in \mathcal{A}_{\mathrm{H}}^{+}$for all $m \in\{1, \ldots, M\}$. Define the associated semialgebraic set

$$
S:=\left\{s \in \mathbb{R}^{N} \mid p_{m}\left(s_{1}, \ldots, s_{N}\right) \geq 0 \text { for all } m \in\{1, \ldots, M\}\right\} .
$$

Then the unital ${ }^{*}$-homomorphism $\mathbb{C}\left[x_{1}, \ldots, x_{N}\right] \ni q \mapsto q\left(a_{1}, \ldots, a_{N}\right) \in \mathcal{A}$ is positive with respect to the $S$-pointwise order on $\mathbb{C}\left[x_{1}, \ldots, x_{N}\right]$. 
Proof: First let $q \in \mathbb{C}\left[x_{1}, \ldots, x_{N}\right]_{\mathrm{H}}$ be given such that $q\left(s_{1}, \ldots, s_{N}\right)>0$ for all $s \in S$. By the Positivstellensatz of Krivine and Stengle, there exist two polynomials

$$
r_{1}, r_{2} \in\left\langle\left\{\prod_{m=1}^{M}\left(p_{m}\right)^{\sigma(m)} \mid \sigma(1), \ldots, \sigma(M) \in\{0,1\}\right\}\right\rangle_{\mathrm{pos}}
$$

such that $\left(1+r_{1}\right) q=1+r_{2}$ (this version of the Positivstellensatz can be obtained from the more traditional formulation $r_{1} q=1+r_{2}$ by the well-known trick of multiplying with $q$ and adding the identities, which yields $\left.\left(1+r_{1}+r_{2}\right) q=1+r_{2}+r_{1} q^{2}\right)$. This shows that $\left(\mathbb{1}+r_{1}\left(a_{1}, \ldots, a_{N}\right)\right) q\left(a_{1}, \ldots, a_{N}\right)=\mathbb{1}+$ $r_{2}\left(a_{1}, \ldots, a_{N}\right)$ and from the previous Corollary 4.3 it follows that $r_{1}\left(a_{1}, \ldots, a_{N}\right), r_{2}\left(a_{1}, \ldots, a_{N}\right) \in \mathcal{A}_{\mathrm{H}}^{+}$, so $q\left(a_{1}, \ldots, a_{N}\right) \in \mathcal{A}_{\mathrm{H}}^{+}$because $\mathcal{A}$ is radical.

For general $q \in \mathbb{C}\left[x_{1}, \ldots, x_{N}\right]_{\mathrm{H}}$ which is $S$-pointwise positive, this shows that $q\left(a_{1}, \ldots, a_{N}\right)+\epsilon \mathbb{1}=$ $(q+\epsilon)\left(a_{1}, \ldots, a_{N}\right) \in \mathcal{A}_{\mathrm{H}}^{+}$for all $\left.\epsilon \in\right] 0, \infty\left[\right.$, and thus $q\left(a_{1}, \ldots, a_{N}\right) \in \mathcal{A}_{\mathrm{H}}^{+}$because $\mathcal{A}$ is Archimedean.

From this proof it also becomes clear that $\mathbb{C}\left[x_{1}, \ldots, x_{N}\right]$ with the algebraic order is not radical for $N \geq 2$, because there exist real polynomials that are (strictly) pointwise positive on whole $\mathbb{R}^{N}$ but not sums of squares, hence not algebraically positive. The first paragraph thus fails for this algebra and $a_{n}:=x_{n}, M:=1, p_{1}:=1$.

In order to construct radical Archimedean ordered *-algebras, it will be helpful to discuss algebras in which many elements are invertible. Recall that a ${ }^{*}$-algebra $\mathcal{A}$ is called symmetric if $a \pm \mathrm{i} \mathbb{1}$ has a multiplicative inverse for all $a \in \mathcal{A}_{\mathrm{H}}$, or equivalently if $\mathbb{1}+a^{2}$ is invertible for all $a \in \mathcal{A}_{\mathrm{H}}$. However, there are also similar, but non-equivalent notions where one demands that e.g. $\mathbb{1}+a^{*} a$ is invertible for all $a \in \mathcal{A}$ or that $\mathbb{1}+\sum_{n=1}^{N} a_{n}^{*} a_{n}$ is invertible for all $a_{1}, \ldots, a_{N} \in \mathcal{A}$ with $N \in \mathbb{N}$, see [7, Chap. 9.8] for a comparison. In ordered ${ }^{*}$-algebras, there is another, even stronger possibility:

Definition 4.5 An ordered ${ }^{*}$-algebra $\mathcal{A}$ is called symmetric if every coercive element of $\mathcal{A}_{\mathrm{H}}$ has a multiplicative inverse.

In order to prove that every symmetric Archimedean ordered *-algebra is radical, we need some preliminary lemmas:

Lemma 4.6 Let $\mathcal{A}$ be an ordered ${ }^{*}$-algebra, $a \in \mathcal{A}_{\mathrm{H}}$ coercive and $\left.\epsilon \in\right] 0, \infty\left[\right.$ such that $a \geq \epsilon \mathbb{1}$, then $a^{-1}$ is Hermitian, positive and uniformly bounded with $\left\|a^{-1}\right\|_{\infty} \leq \epsilon^{-1}$.

Proof: We have $a^{-1}=\left(a a^{-1}\right)^{*} a^{-1}=\left(a^{-1}\right)^{*} a a^{-1} \in \mathcal{A}_{\mathrm{H}}^{+}$, and $a=\epsilon^{-1} a^{2}-\epsilon^{-1}(a-\epsilon \mathbb{1})^{2}-(a-\epsilon \mathbb{1}) \leq$ $\epsilon^{-1} a^{2}$ implies $a^{-1}=a^{-1} a a^{-1} \leq \epsilon^{-1} a^{-1} a^{2} a^{-1}=\epsilon^{-1} \mathbb{1}$ so that $\left\|a^{-1}\right\|_{\infty} \leq \epsilon^{-1}$ by Proposition 3.11.

Lemma 4.7 Let $\mathcal{A}$ be an ordered ${ }^{*}$-algebra and $a \in\left(\mathcal{A}^{\mathrm{bd}}\right)_{\mathrm{H}}^{+}$, then there exist two sequences of polynomials $\left(p_{n}\right)_{n \in \mathbb{N}}$ and $\left(q_{n}\right)_{n \in \mathbb{N}}$ in $\mathbb{R}[x] \cong \mathbb{C}[x]_{\mathrm{H}}$ such that the identity $a+q_{n}(a)=p_{n}^{2}(a)$ and the estimates $0 \leq p_{n}(a)$ and $0 \leq q_{n}(a) \leq \mathbb{1} / n$ hold

Proof: Let $u:=\|a\|_{\infty}+1$ so that $0 \leq a \leq u \mathbb{1}$ by Proposition 3.11. By the (Stone-)Weierstraß theorem, applied to the continuous function $\sqrt{\cdot}:[0, u] \rightarrow \mathbb{R}$, there exists for every $n \in \mathbb{N}$ a polynomial $p_{n}^{\prime} \in$ $\mathbb{C}[x]_{\mathrm{H}}$ such that $\left|\sqrt{t}-p_{n}^{\prime}(t)\right| \leq 1 /(4 n(\sqrt{u}+1))$ holds for all $t \in[0, u]$. Define $p_{n}:=p_{n}^{\prime}+1 /(4 n(\sqrt{u}+1))$ and $q_{n}:=p_{n}^{2}-x$. Then the estimate $0 \leq \sqrt{t} \leq p_{n}(t) \leq \sqrt{t}+1 /(2 n(\sqrt{u}+1))$ and thus $0 \leq p_{n}^{2}(t)-t \leq 1 / n$ hold for all $t \in[0, u]$. 
Using the fundamental theorem of algebra, one can show that every polynomial $r \in \mathbb{C}[x]_{\mathrm{H}}$ which is pointwise positive on $[0, u]$ is an element of $\langle\{x, u-x\}\rangle_{\text {pos }}$, see e.g. [12, Prop. 3.3], hence $r(a) \in \mathcal{A}_{\mathrm{H}}^{+}$. The pointwise estimates for $p_{n}$ and $q_{n}$ thus imply that $0 \leq p_{n}(a)$ and $0 \leq q_{n}(a) \leq \mathbb{1} / n$, and the identity $a+q_{n}(a)=p_{n}(a)^{2}$ is fulfilled by construction.

Proposition 4.8 Every symmetric Archimedean ordered ${ }^{*}$-algebra is radical.

Proof: Let two commuting elements $a, b \in \mathcal{A}_{\mathrm{H}}$ and $\left.\epsilon \in\right] 0, \infty[$ be given such that $a$ is coercive with $a \geq \epsilon \mathbb{1}$ and $a b \geq 0$. Using the previous Lemmas 4.6 and 4.7 one can construct sequences of polynomials $\left(p_{n}\right)_{n \in \mathbb{N}}$ and $\left(q_{n}\right)_{n \in \mathbb{N}}$ such that $a^{-1}+q_{n}\left(a^{-1}\right)=p_{n}\left(a^{-1}\right)^{2}$ with $0 \leq q_{n}\left(a^{-1}\right) \leq \mathbb{1} / n$ for all $n \in \mathbb{N}$, so $0 \leq p_{n}\left(a^{-1}\right) a b p_{n}\left(a^{-1}\right)=b+q_{n}\left(a^{-1}\right) a b$. Using Lemma 3.15 with $\chi=\sqrt{n}$ and that $q_{n}\left(a^{-1}\right)^{2} \leq \mathbb{1} / n^{2}$ by Lemma 3.8, it follows that $2 q_{n}\left(a^{-1}\right) a b \leq \chi^{-2} a^{2} b^{2}+\chi^{2} q_{n}\left(a^{-1}\right)^{2} \leq\left(a^{2} b^{2}+\mathbb{1}\right) / n$. As $\mathcal{A}$ is Archimedean it follows that $0 \leq b$, so $\mathcal{A}$ is radical.

In the uniformly complete case, we will also see that the various notions of symmetric ${ }^{*}$-algebras that were mentioned before are actually equivalent:

Lemma 4.9 Let $\mathcal{A}$ be an ordered ${ }^{*}$-algebra, $a, b \in \mathcal{A}$ and $d \in \mathcal{A}_{\mathrm{H}}^{+}$, then

$$
a^{*} c b+b^{*} c a \leq a^{*} d a+b^{*} d b
$$

holds for all $c \in \mathcal{A}_{\mathrm{H}}$ fulfilling $-d \leq c \leq d$.

Proof: Given $c \in \mathcal{A}_{\mathrm{H}}$ with $-d \leq c \leq d$, then write $c_{(+)}:=(d+c) / 2 \in \mathcal{A}_{\mathrm{H}}^{+}$and $c_{(-)}:=(d-c) / 2 \in \mathcal{A}_{\mathrm{H}}^{+}$. Note that $c=c_{(+)}-c_{(-)}$and $d=c_{(+)}+c_{(-)}$. From $0 \leq(a-b)^{*} c_{(+)}(a-b)$ and $0 \leq(a+b)^{*} c_{(-)}(a+b)$ it follows that

$$
a^{*} c_{(+)} b+b^{*} c_{(+)} a \leq a^{*} c_{(+)} a+b^{*} c_{(+)} b \quad \text { and } \quad-a^{*} c_{(-)} b-b^{*} c_{(-)} a \leq a^{*} c_{(-)} a+b^{*} c_{(-)} b
$$

hold. Adding these two estimates yields (4.2).

Lemma 4.10 Let $\mathcal{A}$ be an Archimedean ordered ${ }^{*}$-algebra, $\hat{a} \in \mathcal{A}_{\mathrm{H}}$ and $\left(a_{n}\right)_{n \in \mathbb{N}}$ a sequence in $\mathcal{A}_{\mathrm{H}}$ of invertible elements such that the sequence of their inverses converges with respect to the uniform metric against some limit $e:=\lim _{n \rightarrow \infty} a_{n}^{-1} \in \mathcal{A}$. Moreover, assume that there exist elements $c, d \in \mathcal{A}_{\mathrm{H}}^{+}$ such that $a_{n}^{2} \leq c$ for all $n \in \mathbb{N}$ and such that for all $\left.\epsilon \in\right] 0, \infty[$ there exists an $N \in \mathbb{N}$ for which $-\epsilon d \leq \hat{a}-a_{n} \leq \epsilon d$ is fulfilled for all $n \in \mathbb{N}$ with $n \geq N$. Then $\hat{a}$ is invertible and $\hat{a}^{-1}=e=\lim _{n \rightarrow \infty} a_{n}^{-1}$.

Proof: As all $a_{n}$ with $n \in \mathbb{N}$ and thus also their inverses $a_{n}^{-1}$ are Hermitian, $e$ is Hermitian by Proposition 3.16. Therefore it is sufficient to show that $\hat{a} e=\mathbb{1}$, which then also implies $e \hat{a}=(\hat{a} e)^{*}=\mathbb{1}$.

So let $\epsilon \in] 0, \infty\left[\right.$ be given, then there exists an $n \in \mathbb{N}$ such that $-\epsilon d \leq \hat{a}-a_{n} \leq \epsilon d$ and $\left\|e-a_{n}^{-1}\right\|_{\infty} \leq \epsilon$ hold, thus also $\left(e-a_{n}^{-1}\right)^{2} \leq \epsilon^{2} \mathbb{1}$ by Proposition 3.12. Using the previous Lemma 4.9 and Lemma 3.15 with $\chi:=1 / \sqrt{\epsilon}$ one finds that

$$
\underbrace{\left(\mathrm{i}^{k} \mathbb{1}\right)^{*}\left(\hat{a}-a_{n}\right) e+e\left(\hat{a}-a_{n}\right)\left(\mathrm{i}^{k} \mathbb{1}\right)}_{\leq \epsilon(d+e d e)}+\underbrace{\left(\mathrm{i}^{k} a_{n}\right)^{*}\left(e-a_{n}^{-1}\right)+\left(e-a_{n}^{-1}\right)\left(\mathrm{i}^{k} a_{n}\right)}_{\leq \epsilon(c+\mathbb{1})} \leq \epsilon(\mathbb{1}+c+d+e d e)
$$


holds for all $k \in\{0,1,2,3\}$, or equivalently, $-\epsilon(\mathbb{1}+c+d+e d e) \leq 2 \operatorname{Re}(\hat{a} e-\mathbb{1}) \leq \epsilon(\mathbb{1}+c+d+e d e)$ and $-\epsilon(\mathbb{1}+c+d+e d e) \leq 2 \operatorname{Im}(\hat{a} e-\mathbb{1}) \leq \epsilon(\mathbb{1}+c+d+e d e)$ because $\hat{a} e-\mathbb{1}=\left(\hat{a}-a_{n}\right) e+a_{n}\left(e-a_{n}^{-1}\right)$

Proposition 4.11 Let $\mathcal{A}$ be a uniformly complete Archimedean ordered ${ }^{*}$-algebra and $a, b \in \mathcal{A}_{\mathrm{H}}^{+}$commuting such that $a$ is coercive, $b$ invertible and $a \leq b^{2}$. Then $a$ is also invertible.

Proof: It is sufficient to show that $\hat{a}:=b a b$ has an inverse, then $a$ is also invertible with $a^{-1}=b \hat{a}^{-1} b$. There is an $\epsilon \in] 0, \infty\left[\right.$ such that $\epsilon \mathbb{1} \leq a$, and consequently $\epsilon \mathbb{1} \leq b^{2}$ and $\epsilon^{2} \mathbb{1} \leq \epsilon b^{2} \leq \hat{a} \leq b^{4}$ hold.

Define $a_{n}:=\hat{a}+b^{4} / n$ for all $n \in \mathbb{N}, c:=\hat{a}^{2}+3 b^{8}$ and $d:=b^{4}$, then $a_{n}^{2}=\hat{a}^{2}+2 b^{2} \hat{a} b^{2} / n+b^{8} / n^{2} \leq c$ and $-d / n=\hat{a}-a_{n} \leq d / n$. In order to apply the previous Lemma 4.10 it only remains to show that all $a_{n}$ with $n \in \mathbb{N}$ are invertible and that the sequence of their inverses is a Cauchy sequence.

Consider $b^{-2} a_{n} b^{-2}=b^{-2} \hat{a} b^{-2}+\mathbb{1} / n$. Then $\mathbb{1} / n \leq b^{-2} a_{n} b^{-2} \leq(1+1 / n) \mathbb{1}$, so $b^{-2} a_{n} b^{-2}$ is a coercive element of $\mathcal{A}^{\text {bd }}$. From Proposition 3.16 it follows that $\mathcal{A}^{\text {bd }}$ is uniformly complete itself, hence a $C^{*}$-algebra, so $b^{-2} a_{n} b^{-2}$ is invertible in $\mathcal{A}^{\text {bd }}$ (the inverse can be constructed explicitly e.g. using a Neumann series). Consequently, $a_{n}$ is also invertible with $a_{n}^{-1}=b^{-2}\left(b^{-2} a_{n} b^{-2}\right)^{-1} b^{-2}$. Moreover, using $-\left|m^{-1}-n^{-1}\right| b^{4} \leq a_{n}-a_{m} \leq\left|m^{-1}-n^{-1}\right| b^{4}$ and Lemma 4.9, one obtains the estimate

$$
a_{m}^{-1}-a_{n}^{-1}=\frac{a_{m}^{-1}\left(a_{n}-a_{m}\right) a_{n}^{-1}+a_{n}^{-1}\left(a_{n}-a_{m}\right) a_{m}^{-1}}{2} \leq\left|\frac{1}{m}-\frac{1}{n}\right| \frac{a_{m}^{-1} b^{4} a_{m}^{-1}+a_{n}^{-1} b^{4} a_{n}^{-1}}{2}
$$

for all $m, n \in \mathbb{N}$. From $\epsilon b^{2} \leq \hat{a}$ it follows that $\epsilon \mathbb{1} \leq b^{-1} \hat{a} b^{-1}$ and thus

$$
\epsilon^{2} b^{4} \leq\left(\hat{a}-\epsilon b^{2}\right)^{2}+\epsilon^{2} b^{4}=\hat{a}^{2}-2 \epsilon b\left(\hat{a}-\epsilon b^{2}\right) b \leq \hat{a}^{2} \leq \hat{a}^{2}+2 b^{2} \hat{a} b^{2} / n+b^{8} / n^{2}=a_{n}^{2}
$$

for all $n \in \mathbb{N}$. The combination of these estimates yields $a_{m}^{-1}-a_{n}^{-1} \leq \epsilon^{-2}\left|m^{-1}-n^{-1}\right| \mathbb{1}$ for all $m, n \in \mathbb{N}$, so $\left(a_{n}^{-1}\right)_{n \in \mathbb{N}}$ is indeed a Cauchy sequence.

Corollary 4.12 Let $\mathcal{A}$ be a uniformly complete Archimedean ordered ${ }^{*}$-algebra in which $\mathbb{1}+a^{2}$ is invertible for all $a \in \mathcal{A}_{\mathrm{H}}$, then $\mathcal{A}$ is symmetric.

Proof: Given a coercive $a \in \mathcal{A}_{\mathrm{H}}$, then one can apply the previous Proposition 4.11 with $b:=\mathbb{1}+a^{2}$ because $a \leq 2 a=\mathbb{1}+a^{2}-(\mathbb{1}-a)^{2} \leq \mathbb{1}+a^{2} \leq\left(\mathbb{1}+a^{2}\right)^{2}$.

\section{$5 \quad \Phi^{*}$-Algebras}

It has already been mentioned in Section 2 that Riesz spaces which carry a non-commutative multiplication have rather pathological properties. Because of this, a well-behaved non-commutative generalization of the notion of $\Phi$-algebras must necessarily deal with some restrictions to the infima and suprema. Moreover, like in $\Phi$-algebras, there should also be a compatibility between suprema, infima and the product, but it might not be immediately clear what exactly this compatibility should be. The following observation, which gives a mostly algebraic characterization of suprema and infima, might serve as a motivation (recall that.${ }^{\prime}$ denotes the commutant):

Proposition 5.1 Let $\mathcal{A}$ be a radical Archimedean ordered ${ }^{*}$-algebra, a,b $\in \mathcal{A}_{\mathrm{H}}$ commuting and let $x \in\{a, b\}^{\prime \prime} \cap \mathcal{A}_{\mathrm{H}}$ be such that $x^{2}+a b=x(a+b)$, then the following holds: 
- If $2 x \geq a+b$, then $x$ is the supremum of $a$ and $b$ in $\{a, b\}^{\prime} \cap \mathcal{A}_{\mathrm{H}}$.

- If $2 x \leq a+b$, then $x$ is the infimum of $a$ and $b$ in $\{a, b\}^{\prime} \cap \mathcal{A}_{\mathrm{H}}$.

Proof: First assume that $2 x \geq a+b$, then $(2 x-a-b)^{2}=4 x^{2}-4 x(a+b)+4 a b+(a-b)^{2}=(a-b)^{2}$ implies $-(2 x-a-b) \leq a-b \leq 2 x-a-b$ by Proposition 4.2 , so $x \geq a$ and $x \geq b$. Moreover, if some $y \in\{a, b\}^{\prime} \cap \mathcal{A}_{\mathrm{H}}$ also fulfils $y \geq a$ and $y \geq b$, then $0 \leq(y-a)(y-b)=y^{2}-y(a+b)+a b$ by Corollary 4.3 and thus $(2 y-a-b)^{2}=4 y^{2}-4 y(a+b)+4 a b+(a-b)^{2} \geq(a-b)^{2}=(2 x-a-b)^{2}$. Proposition 4.2 now shows that $2 y-a-b \geq 2 x-a-b$, so $y \geq x$ and $x$ is indeed the supremum of $a$ and $b$ in $\{a, b\}^{\prime} \cap \mathcal{A}_{\mathrm{H}}$. If $2 x \leq a+b$, then one can apply the above argument to $-x,-a$ and $-b$.

The following definition thus makes sense and describes suprema and infima that fulfil additional algebraic conditions:

Definition 5.2 Let $\mathcal{A}$ be a radical Archimedean ordered ${ }^{*}$-algebra and $a, b \in \mathcal{A}_{\mathrm{H}}$ commuting. Then $a \vee b$ is (if it exists) the element in $\{a, b\}^{\prime \prime} \cap \mathcal{A}_{\mathrm{H}}$ which fulfils

$$
2(a \vee b) \geq a+b \quad \text { and } \quad(a \vee b)^{2}+a b=(a \vee b)(a+b)
$$

Similarly, $a \wedge b$ is (if it exists) the element in $\{a, b\}^{\prime \prime} \cap \mathcal{A}_{\mathrm{H}}$ which fulfils

$$
2(a \wedge b) \leq a+b \quad \text { and } \quad(a \wedge b)^{2}+a b=(a \wedge b)(a+b) .
$$

$A$ radical Archimedean ordered ${ }^{*}$-algebra $\mathcal{A}$ in which $a \vee b$ and $a \wedge b$ exist for all commuting $a, b \in \mathcal{A}_{\mathrm{H}}$ will be called a $\underline{\Phi^{*} \text {-algebra }}$.

Proposition 5.1 especially guarantees that $a \vee b$ and $a \wedge b$ (if they exist) are uniquely determined as certain suprema and infima. In a $\Phi^{*}$-algebra $\mathcal{A}$, Proposition 5.1 also has a rather trivial, but noteworthy converse: If $a, b \in \mathcal{A}_{\mathrm{H}}$ commute and $x \in\{a, b\}^{\prime} \cap \mathcal{A}_{\mathrm{H}}$ is the supremum or infimum of $a$ and $b$ in some real linear subspace $V$ of $\mathcal{A}_{\mathrm{H}}$ such that $\{a, b\}^{\prime \prime} \cap \mathcal{A}_{\mathrm{H}} \subseteq V \subseteq\{a, b\}^{\prime} \cap \mathcal{A}_{\mathrm{H}}$, then $x$ coincides with $a \vee b$ or $a \wedge b$, respectively, due to the uniqueness of the suprema and infima; so especially $x \in\{a, b\}^{\prime \prime} \cap \mathcal{A}_{\mathrm{H}}$ and $x^{2}+a b=x(a+b)$.

There are some basic results about these suprema and infima which are not very surprising as they mostly mimic the rules in ordered vector spaces, and which can easily be checked:

Proposition 5.3 Let $\mathcal{A}$ be a radical Archimedean ordered ${ }^{*}$-algebra and $a, b \in \mathcal{A}_{\mathrm{H}}$ commuting. Then $a \vee b$ exists if and only if $a \wedge b$ exists, and the two are related by

$$
(a \vee b)+(a \wedge b)=a+b \quad \text { and } \quad(a \vee b)(a \wedge b)=a b
$$

Moreover, if one, hence both of $a \vee b$ and $a \wedge b$ exist, then the following holds:

i.) $b \vee a=a \vee b$ and $b \wedge a=a \wedge b$ exist.

ii.) $(\lambda a) \vee(\lambda b)=\lambda(a \vee b)$ and $(\lambda a) \wedge(\lambda b)=\lambda(a \wedge b)$ exist for all $\lambda \in[0, \infty[$.

iii.) $(-a) \wedge(-b)=-(a \vee b)$ and $(-a) \vee(-b)=-(a \wedge b)$ exist. 
iv.) $(a+c) \vee(b+c)=(a \vee b)+c$ exists for all $c \in\{a, b\}^{\prime} \cap \mathcal{A}_{\mathrm{H}}$ with $(a \vee b)+c \in\{a+c, b+c\}^{\prime \prime}$, and $(a+c) \wedge(b+c)=(a \wedge b)+c$ exists for all $c \in\{a, b\}^{\prime} \cap \mathcal{A}_{\mathrm{H}}$ with $(a \wedge b)+c \in\{a+c, b+c\}^{\prime \prime}$.

Proof: If $x \in\{a, b\}^{\prime \prime} \cap \mathcal{A}_{\mathrm{H}}$ fulfils $x^{2}+a b=x(a+b)$, then also $y:=a+b-x \in\{a, b\}^{\prime \prime} \cap \mathcal{A}_{\mathrm{H}}$ fulfils $y^{2}+a b=y(a+b)$. Especially using $x=a \vee b$ and $x=a \wedge b$ it follows that $a \vee b$ exists if and only if $a \wedge b$ exists and that $(a \vee b)+(a \wedge b)=a+b$. As a consequence, $(a \vee b)(a \wedge b)=(a \vee b)(a+b)-(a \vee b)^{2}=a b$. Checking that i.), ii.) and iii.) hold is easy and for part $i v$.) one essentially only needs to verify that

$$
\begin{aligned}
((a \vee b)+c)^{2}+(a+c)(b+c) & =(a \vee b)^{2}+a b+2 c(a \vee b)+(a+b+2 c) c \\
& =(a+b)(a \vee b)+2 c(a \vee b)+(a+b+2 c) c \\
& =(a+b+2 c)((a \vee b)+c)
\end{aligned}
$$

With respect to part iv.) we note that the conditions $(a \vee b)+c,(a \wedge b)+c \in\{a+c, b+c\}^{\prime \prime}$ are superfluous if it is a priori known that $(a+c) \vee(b+c)$ and $(a+c) \wedge(b+c)$ exist, i.e. especially if $\mathcal{A}$ is a $\Phi^{*}$-algebra. This is due to the observation that $(a \vee b)+c$ and $(a \wedge b)+c$ with $c \in\{a, b\}^{\prime} \cap \mathcal{A}_{\mathrm{H}}$ are the supremum and infimum, respectively, of $a+c$ and $b+c$ in $V:=\{a, b\}^{\prime} \cap\{a+c, b+c\}^{\prime} \cap \mathcal{A}_{\mathrm{H}}$, so the discussion under Definition 5.2 applies.

One important special case of these suprema in ordered ${ }^{*}$-algebras are absolute values:

Definition 5.4 Let $\mathcal{A}$ be a radical Archimedean ordered ${ }^{*}$-algebra and $a \in \mathcal{A}_{\mathrm{H}}$, then the absolute value of $a$ is defined (if it exists) as the element $|a|:=a \vee(-a)$. If the absolute value exists, then one also defines the $\underline{\text { positive part }} a_{+}:=\frac{1}{2}(|a|+a)$ and the $\underline{\text { negative part }} a_{-}:=\frac{1}{2}(|a|-a)$ of $a$.

Clearly, $|-a|=|a|$ if $|a|$ exists. By definition, the absolute value of an element $a \in \mathcal{A}_{\mathrm{H}}$ is (if it exists) the element $|a| \in\{a\}^{\prime \prime} \cap \mathcal{A}_{\mathrm{H}}^{+}$that fulfils $|a|^{2}=a^{2}$. The earlier results about suprema and infima now show that, like for Riesz spaces, the existence of all absolute values already implies the existence of all suprema and infima of commuting Hermitian elements:

Proposition 5.5 Let $\mathcal{A}$ be a radical Archimedean ordered ${ }^{*}$-algebra and $a, b \in \mathcal{A}_{\mathrm{H}}$ commuting and such that $|a-b|$ exists. Then $a \vee b$ and $a \wedge b$ exist and are given by

$$
a \vee b=\frac{a+b+|a-b|}{2} \quad \text { and } \quad a \wedge b=\frac{a+b-|a-b|}{2} .
$$

Proof: This is just an application of Proposition 5.3 using that $|a-b| \in\{a, b\}^{\prime \prime}$.

As immediate consequences of Propositions 5.3 and 5.5 we obtain:

Corollary 5.6 Let $\mathcal{A}$ be a radical Archimedean ordered ${ }^{*}$-algebra and $a \in \mathcal{A}_{\mathrm{H}}$ such that $|a|$ exists. Then $a \vee 0=a_{+} \in A_{\mathrm{H}}^{+}$and $(-a) \vee 0=-(a \wedge 0)=a_{-} \in A_{\mathrm{H}}^{+}$exist and fulfil $a_{+}+a_{-}=a$ and $a_{-} a_{+}=a_{+} a_{-}=0$.

Corollary 5.7 Let $\mathcal{A}$ be a radical Archimedean ordered ${ }^{*}$-algebra, then $\mathcal{A}$ is a $\Phi^{*}$-algebra if and only if $|a|$ exists for all $a \in \mathcal{A}_{\mathrm{H}}$.

One motivation to study $\Phi^{*}$-algebras is that they indeed are a non-commutative generalization of $\Phi$-algebras: 
Proposition 5.8 Let $\mathcal{R}$ be a $\Phi$-algebra, then its complexification $\mathcal{A}:=\mathcal{R} \otimes \mathbb{C}$ with ${ }^{*}$-involution and multiplication defined by $(r \otimes \lambda)^{*}:=r \otimes \bar{\lambda}$ and $(r \otimes \lambda)(s \otimes \mu):=r s \otimes \lambda \mu$ for all $r, s \in \mathcal{R}$ and all $\lambda, \mu \in \mathbb{C}$ is a commutative $\Phi^{*}$-algebra. Conversely, if $\mathcal{A}$ is a commutative $\Phi^{*}$-algebra, then its real unital subalgebra $\mathcal{A}_{\mathrm{H}}$ is a $\Phi$-algebra.

Proof: First let $\mathcal{R}$ be a $\Phi$-algebra and $\mathcal{A}:=\mathcal{R} \otimes \mathbb{C}$. Then it is clear that $\mathcal{A}$ is a commutative Archimedean ordered *-algebra, and it is also radical: Given two commuting $a, b \in \mathcal{A}_{\mathrm{H}} \cong \mathcal{R}$ such that $a \geq \epsilon \mathbb{1}$ for some $\epsilon \in] 0, \infty\left[\right.$ and $0 \leq a b$, then write $b_{+}:=\sup \{b, 0\}$ and $b_{-}:=\sup \{-b, 0\}$. Note that it is not yet clear that $b_{+}$and $b_{-}$are the positive and negative part of $b$ like in Definition 5.4, but it follows from the general calculation rules in Riesz spaces and $\Phi$-algebras that $b=b_{+}-b_{-}$and $b_{+} b_{-}=0$. Consequently, $0 \leq b_{-} a b=-b_{-} a b_{-} \leq-\epsilon\left(b_{-}\right)^{2} \leq 0$, so $\left(b_{-}\right)^{2}=0$. Proposition 3.9 now shows that $b_{-}=0$ and therefore $b=b_{+} \geq 0$. As the order-theoretic absolute value $|a|:=\sup \{a,-a\} \in \mathcal{A}_{\mathrm{H}}^{+}$of any $a \in \mathcal{A}_{\mathrm{H}}$ indeed fulfils $|a|^{2}=a^{2}$ by the calculation rules in $\Phi$-algebras, it also describes the absolute value as in Definition 5.4 and therefore $\mathcal{A}$ is a $\Phi^{*}$-algebra by the previous Corollary 5.7.

Now let $\mathcal{A}$ be an arbitrary commutative $\Phi^{*}$-algebra. Then $\mathcal{A}_{\mathrm{H}}$ is a real commutative unital associative algebra and a Riesz space by Proposition 5.1. Corollary 4.3 shows that $a b \in \mathcal{A}_{\mathrm{H}}^{+}$for all $a, b \in \mathcal{A}_{\mathrm{H}}^{+}$. Given $a, b, c \in \mathcal{A}_{\mathrm{H}}^{+}$with $\inf \{a, b\}=0$, then $a \wedge b=\inf \{a, b\}=0$ and thus $a b=0$. It follows that $0 \leq(a c \wedge b)^{2} \leq(a c \wedge b)(a c \vee b)=a c b=0$ holds by Corollary 4.3 and Proposition 5.3, so $(a c \wedge b)^{2}=0$. Proposition 3.9 now shows that $a c \wedge b=0$ and thus $\mathcal{A}_{\mathrm{H}}$ is a $\Phi$-algebra.

Non-commutative examples of $\Phi^{*}$-algebras will be described in Sections 7 and 8 .

Another interesting observation about $\Phi^{*}$-algebras is that injective positive unital *-homomorphisms between them are automatically order embeddings. This is roughly similar to the case of *algebras endowed with a Fréchet-topology, where surjective continuous linear maps are automatically open by the open mapping theorem:

Proposition 5.9 Let $\Psi: \mathcal{A} \rightarrow \mathcal{B}$ be an injective positive unital ${ }^{*}$-homomorphism from a $\Phi^{*}$-algebra $\mathcal{A}$ to an ordered ${ }^{*}$-algebra $\mathcal{B}$, then $\Psi$ is automatically an order embedding.

Proof: Let $a \in \mathcal{A}_{\mathrm{H}}$ with $\Psi(a) \geq 0$ be given. Then $a=a_{+}-a_{-}$with $a_{+}, a_{-} \in \mathcal{A}_{\mathrm{H}}^{+}$and $a_{+} a_{-}=0$ by Corollary 5.6, so on the one hand $\left(a_{-}\right)^{3} \geq 0$ implies $\Psi\left(a_{-}\right)^{3} \geq 0$, and on the other, $\left(a_{-}\right)^{3}=-\left(a_{-} a a_{-}\right)$ implies $\Psi\left(a_{-}\right)^{3}=-\Psi\left(a_{-}\right) \Psi(a) \Psi\left(a_{-}\right) \leq 0$, so $\Psi\left(a_{-}\right)^{3}=0$. As $\Psi$ is injective, it follows that $\left(a_{-}\right)^{3}=0$ and thus $a_{-}=0$ by Proposition 3.9. So $a=a_{+} \geq 0$ and $\Psi$ is an order embedding.

Moreover, positive unital *-homomorphisms between $\Phi^{*}$-algebras are compatible with $\vee, \wedge$ and the absolute value:

Lemma 5.10 Let $\mathcal{A}$ be a radical Archimedean ordered ${ }^{*}$-algebra and $a \in \mathcal{A}_{\mathrm{H}}^{+}, b \in\{a\}^{\prime \prime} \cap \mathcal{A}_{\mathrm{H}}^{+}$and $c \in\{a\}^{\prime} \cap \mathcal{A}_{\mathrm{H}}^{+}$such that $b^{2}=c^{2}$, then $b=c$ and especially $c \in\{a\}^{\prime \prime}$.

Proof: Note that $b$ and $c$ commute, so $b^{2}=c^{2}$ implies $b=c$ by Proposition 4.2.

Proposition 5.11 Let $\Psi: \mathcal{A} \rightarrow \mathcal{B}$ be a positive unital ${ }^{*}$-homomorphism between two $\Phi^{*}$-algebras $\mathcal{A}, \mathcal{B}$ and $a, \tilde{a} \in \mathcal{A}_{\mathrm{H}}$ commuting. Then

$$
\Psi(a) \vee \Psi(\tilde{a})=\Psi(a \vee \tilde{a}), \quad \Psi(a) \wedge \Psi(\tilde{a})=\Psi(a \wedge \tilde{a}) \quad \text { and } \quad|\Psi(a)|=\Psi(|a|)
$$


hold.

Proof: As $\vee$ and $\wedge$ can be expressed using the absolute value, it is enough to show that $|\Psi(a)|=\Psi(|a|)$ holds. It is easy to check that $\Psi(|a|) \in\{\Psi(a)\}^{\prime} \cap \mathcal{B}_{\mathrm{H}}^{+}$and that $\Psi(|a|)^{2}=\Psi(a)^{2}$. As it is already known that $|\Psi(a)| \in\{\Psi(a)\}^{\prime \prime} \cap \mathcal{B}_{\mathrm{H}}^{+}$exists, it follows from the previous Lemma 5.10 that $|\Psi(a)|=\Psi(|a|)$.

As a last result we note that in the uniformly complete case, the existence of infima is helpful for the construction of a multiplicative inverse:

Lemma 5.12 Let $\mathcal{A}$ be a radical Archimedean ordered ${ }^{*}$-algebra, $a \in \mathcal{A}_{\mathrm{H}}$ and $\left.\lambda \in\right] 0, \infty[$. If $a \wedge \lambda \mathbb{1}$ exists, then it fulfils the estimate $a \leq(a \wedge \lambda \mathbb{1})+a^{2} /(4 \lambda)$.

Proof: As $a$ and $a \wedge \lambda \mathbb{1}$ commute, $a(a \wedge \lambda \mathbb{1})=(a(a \wedge \lambda \mathbb{1})+(a \wedge \lambda \mathbb{1}) a) / 2 \leq a^{2} / 4+(a \wedge \lambda \mathbb{1})^{2}$ holds by Lemma 3.15 with $\chi:=\sqrt{2}$. From $(a \wedge \lambda \mathbb{1})^{2}+\lambda a=(a+\lambda \mathbb{1})(a \wedge \lambda \mathbb{1})$ it now follows that $(a \wedge \lambda \mathbb{1})^{2}+\lambda a \leq a^{2} / 4+(a \wedge \lambda \mathbb{1})^{2}+\lambda(a \wedge \lambda \mathbb{1})$.

Lemma 5.13 Let $\mathcal{A}$ be a radical Archimedean ordered ${ }^{*}$-algebra and $a, b \in \mathcal{A}_{\mathrm{H}}^{+}$with $a \leq b$ commuting and invertible, then $a^{-1} \geq b^{-1}$.

Proof: As $b-a, a^{-1}, b^{-1} \in\{a, b\}^{\prime \prime} \cap \mathcal{A}_{\mathrm{H}}^{+}$are pairwise commuting, their product $a^{-1}(b-a) b^{-1}=a^{-1}-b^{-1}$ is positive by Corollary 4.3 .

Proposition 5.14 Let $\mathcal{A}$ be a radical and uniformly complete Archimedean ordered *-algebra and let $a \in \mathcal{A}_{\mathrm{H}}$ be a coercive element for which $a \wedge n \mathbb{1}$ exists for all $n \in \mathbb{N}$, then a is invertible.

Proof: Proposition 5.1 shows that $a \wedge n \mathbb{1}$ is, for every $n \in \mathbb{N}$, the infimum of $a$ and $n \mathbb{1}$ in the commutative real unital subalgebra $\{a\}^{\prime \prime} \cap \mathcal{A}_{\mathrm{H}}$ of $\mathcal{A}$. As $a$ is coercive, there exists $\left.\left.\epsilon \in\right] 0,1\right]$ such that $\epsilon \mathbb{1} \leq a$, and then $\epsilon \mathbb{1} \leq a \wedge n \mathbb{1} \leq n \mathbb{1}$ shows that $a \wedge n \mathbb{1}$ is a coercive element of $\mathcal{A}^{\text {bd }}$. By Proposition 3.16, $\mathcal{A}^{\text {bd }}$ is uniformly complete itself, hence a $C^{*}$-algebra, and thus $a \wedge n \mathbb{1}$ is invertible.

For fixed $m, n \in \mathbb{N}$ with $n \leq m$, the estimate $a \wedge n \mathbb{1} \leq a \wedge m \mathbb{1}$ yields $(a \wedge m \mathbb{1})^{-1} \leq(a \wedge n \mathbb{1})^{-1}$ by the previous Lemma 5.13. Moreover, let $b:=(a \wedge m \mathbb{1})^{-1}+\mathbb{1} / n \in\{a\}^{\prime \prime} \cap \mathcal{A}_{\mathrm{H}}$, then $b$ is also a coercive element of $\mathcal{A}^{\text {bd }}$, hence invertible. From $\mathbb{1} / n \leq b$ and $(a \wedge m \mathbb{1})^{-1} \leq b$ it follows that $b^{-1} \leq n \mathbb{1}$ and $b^{-1} \leq a \wedge m \mathbb{1}$ by the previous Lemma 5.13. So $b^{-1} \leq a \wedge n \mathbb{1}$ and therefore $(a \wedge n \mathbb{1})^{-1} \leq b$ by Lemma 5.13 again. Altogether, this shows that $(a \wedge m \mathbb{1})^{-1} \leq(a \wedge n \mathbb{1})^{-1} \leq(a \wedge m \mathbb{1})^{-1}+\mathbb{1} / n$ for all $m, n \in \mathbb{N}$ with $n \leq m$, so $\mathbb{N} \ni n \mapsto(a \wedge n \mathbb{1})^{-1} \in \mathcal{A}_{\mathrm{H}}$ is a Cauchy sequence with respect to the uniform metric, and thus converges.

From $0 \leq(a \wedge n \mathbb{1}) \leq a$ it follows that $(a \wedge n \mathbb{1})^{2} \leq a^{2}$ by Proposition 4.2 and that $0 \leq a-(a \wedge n \mathbb{1})$, and Lemma 5.12 shows that $a-(a \wedge n \mathbb{1}) \leq a^{2} /(4 n)$. So one can apply Lemma 4.10 to the sequence $(a \wedge n \mathbb{1})_{n \in \mathbb{N}}$ with $c:=a^{2}$ and $d:=a^{2} / 4$, which shows that $a$ is invertible and $a^{-1}=\lim _{n \rightarrow \infty}(a \wedge n \mathbb{1})^{-1} \cdot \square$

\section{Square Roots}

The usual way to construct absolute values is via square roots of the square. In order to guarantee the uniqueness of the square roots, it makes sense to discuss square roots only in radical Archimedean ordered *-algebras, in which Lemma 5.10 applies: 
Definition 6.1 Let $\mathcal{A}$ be a radical Archimedean ordered ${ }^{*}$-algebra and $a \in \mathcal{A}_{\mathrm{H}}^{+}$. The square root of a is (if it exists) the unique element $\sqrt{a} \in\{a\}^{\prime \prime} \cap \mathcal{A}_{\mathrm{H}}^{+}$fulfilling $\sqrt{a}^{2}=a$.

Proposition 6.2 Let $\mathcal{A}$ be a radical Archimedean ordered ${ }^{*}$-algebra and $a \in \mathcal{A}_{\mathrm{H}}$ and assume that $\sqrt{a^{2}}$ exists, then $|a|$ exists and is given by $|a|=\sqrt{a^{2}}$.

Proof: Note that $\{a\}^{\prime} \subseteq\left\{a^{2}\right\}^{\prime}$, therefore $\left\{a^{2}\right\}^{\prime \prime} \subseteq\{a\}^{\prime \prime}$. So $\sqrt{a^{2}} \in\left\{a^{2}\right\}^{\prime \prime} \cap \mathcal{A}_{\mathrm{H}}^{+} \subseteq\{a\}^{\prime \prime} \cap \mathcal{A}_{\mathrm{H}}^{+}$and ${\sqrt{a^{2}}}^{2}=a^{2}$ show that $|a|=\sqrt{a^{2}}$ exists.

Especially if $\sqrt{a}$ exists for all positive Hermitian elements $a$ of a radical Archimedean ordered *-algebra $\mathcal{A}$, then $\mathcal{A}$ is a $\Phi^{*}$-algebra and $\mathcal{A}_{\mathrm{H}}^{+}=\mathcal{A}_{\mathrm{H}}^{++}$, thus every unital ${ }^{*}$-homomorphism $\Psi: \mathcal{A} \rightarrow \mathcal{B}$ to another ordered ${ }^{*}$-algebra $\mathcal{B}$ is automatically positive as $\Psi(a)=\Psi(\sqrt{a})^{2} \in \mathcal{B}_{\mathrm{H}}^{+}$. On such algebras, the order is even uniquely determined, a result that generalizes the uniqueness of the norm of $C^{*}$-algebras:

Proposition 6.3 Let $\mathcal{A}$ be a radical Archimedean ordered ${ }^{*}$-algebra in which $\sqrt{a}$ exists for all a $\in \mathcal{A}_{\mathrm{H}}^{+}$, then the order on $\mathcal{A}_{\mathrm{H}}$ is uniquely determined in the following sense: Denote the order on $\mathcal{A}_{\mathrm{H}}$ by $\leq$, as always. If $\preccurlyeq$ is any order on $\mathcal{A}_{\mathrm{H}}$ such that $\mathcal{A}$ with $\preccurlyeq$ is an ordered ${ }^{*}$-algebra, then $\leq$ and $\preccurlyeq$ coincide.

Proof: Consider the injective unital ${ }^{*}$-homomorphism id $\mathcal{A}_{\mathcal{A}}$ as a map from $\mathcal{A}$ with $\leq$ to $\mathcal{A}$ with $\preccurlyeq$. Then $\operatorname{id}_{\mathcal{A}}$ is automatically positive due to the existence of square roots, and as $\mathcal{A}$ with $\leq$ is a $\Phi^{*}$-algebra by the previous Proposition 6.2 and Corollary 5.7, Proposition 5.9 applies and shows that $\mathrm{id}_{\mathcal{A}}$ is even an order embedding, i.e. that $\leq$ and $\preccurlyeq$ coincide.

Moreover, unital *-homomorphisms between such algebras are compatible with square roots:

Proposition 6.4 Let $\mathcal{A}$ and $\mathcal{B}$ be two radical Archimedean ordered ${ }^{*}$-algebras and assume that the square roots of all positive Hermitian elements in $\mathcal{A}$ and $\mathcal{B}$ exist. Moreover, let $\Psi: \mathcal{A} \rightarrow \mathcal{B}$ be an (automatically positive) unital ${ }^{*}$-homomorphism. Then $\Psi(\sqrt{a})=\sqrt{\Psi(a)}$ holds for all $a \in \mathcal{A}_{\mathrm{H}}^{+}$.

Proof: Like Proposition 5.11, this follows from Lemma 5.10 because $\Psi(\sqrt{a}) \in\{\Psi(a)\}^{\prime} \cap \mathcal{B}_{\mathrm{H}}^{+}$fulfils $\Psi(\sqrt{a})^{2}=\Psi(a)$ and because it is already known that $\sqrt{\Psi(a)} \in\{\Psi(a)\}^{\prime \prime} \cap \mathcal{B}_{\mathrm{H}}^{+}$exists.

In the uniformly complete case, square roots can oftentimes be explicitly constructed:

Lemma 6.5 Let $\mathcal{A}$ be a radical and uniformly complete Archimedean ordered ${ }^{*}$-algebra, $\hat{a} \in \mathcal{A}_{\mathrm{H}}^{+}$and $\left(a_{n}\right)_{n \in \mathbb{N}}$ a sequence in $\{\hat{a}\}^{\prime \prime} \cap \mathcal{A}_{\mathrm{H}}^{+}$with limit $\hat{a}$. If a sequence $\left(b_{n}\right)_{n \in \mathbb{N}}$ in $\{\hat{a}\}^{\prime \prime} \cap \mathcal{A}_{\mathrm{H}}^{+}$fulfils $b_{n}^{2}=a_{n}$ for all $n \in \mathbb{N}$ and is bounded from above by some $c \in \mathcal{A}_{\mathrm{H}}^{+}$, then $\sqrt{\hat{a}}$ exists and $\sqrt{\hat{a}}=\lim _{n \rightarrow \infty} b_{n}$.

Proof: Given $\epsilon \in] 0,1]$, then there exists an $N \in \mathbb{N}$ such that $-\epsilon^{2} \mathbb{1} \leq a_{n}-a_{N} \leq \epsilon^{2} \mathbb{1}$ holds for all $n \in \mathbb{N}$ with $n \geq N$, hence $b_{N}^{2} \leq b_{n}^{2}+\epsilon^{2} \mathbb{1}$ and $b_{n}^{2} \leq b_{N}^{2}+\epsilon^{2} \mathbb{1}$. This implies $b_{N}^{2} \leq\left(b_{n}+\epsilon \mathbb{1}\right)^{2}$ and $b_{n}^{2} \leq\left(b_{N}+\epsilon \mathbb{1}\right)^{2}$, so $b_{N} \leq b_{n}+\epsilon \mathbb{1}$ and $b_{n} \leq b_{N}+\epsilon \mathbb{1}$ by Proposition 4.2 , or equivalently $-\epsilon \mathbb{1} \leq b_{n}-b_{N} \leq \epsilon \mathbb{1}$. The sequence $\left(b_{n}\right)_{n \in \mathbb{N}}$ thus is a Cauchy sequence and has a limit $\hat{b}:=\lim _{n \rightarrow \infty} b_{n} \in\{\hat{a}\}^{\prime \prime} \cap \mathcal{A}_{\mathrm{H}}^{+}$as $\{\hat{a}\}^{\prime \prime} \cap \mathcal{A}_{\mathrm{H}}^{+}$ is a closed subset of the complete metric space $\mathcal{A}$ by Propositions 3.16.

It only remains to show that $\hat{b}^{2}=\hat{a}$, then $\sqrt{\hat{a}}=\hat{b}$ exists. For all $\left.\left.\epsilon \in\right] 0,1\right]$ there exists an $N \in \mathbb{N}$ such that $-\epsilon \mathbb{1} \leq b_{n}-\hat{b} \leq \epsilon \mathbb{1}$ and $-\epsilon \mathbb{1} \leq a_{n}-\hat{a} \leq \epsilon \mathbb{1}$ hold for all $n \in \mathbb{N}$ with $n \geq N$. The first estimate gives $\hat{b} \leq b_{n}+\epsilon \mathbb{1}$ and $b_{n} \leq \hat{b}+\epsilon \mathbb{1}$, and using Proposition 4.2 one obtains $\hat{b}^{2} \leq\left(b_{n}+\epsilon \mathbb{1}\right)^{2} \leq$ 
$b_{n}^{2}+\epsilon\left(2 b_{n}+\mathbb{1}\right) \leq a_{n}+\epsilon(2 c+\mathbb{1})$ and $a_{n}=b_{n}^{2} \leq(\hat{b}+\epsilon \mathbb{1})^{2} \leq \hat{b}^{2}+\epsilon(2 \hat{b}+\mathbb{1})$. Together with the second estimate this yields $\hat{b}^{2}-\hat{a}=\hat{b}^{2}-a_{n}+a_{n}-\hat{a} \leq \epsilon(2 c+2 \mathbb{1})$ and $\hat{a}-\hat{b}^{2}=\hat{a}-a_{n}+a_{n}-\hat{b}^{2} \leq \epsilon(2 \hat{b}+2 \mathbb{1})$, so $\hat{a}=\hat{b}^{2}$ because $\mathcal{A}$ is Archimedean.

Proposition 6.6 Let $\mathcal{A}$ be a radical and uniformly complete Archimedean ordered ${ }^{*}$-algebra and let $a \in \mathcal{A}_{\mathrm{H}}^{+}$. If additionally $a$ is uniformly bounded, or $a+\mathbb{1} / n$ invertible for all $n \in \mathbb{N}$, then $\sqrt{a}$ exists.

Proof: As $\mathcal{A}^{\text {bd }}$ is a $C^{*}$-algebra due to the completeness of $\mathcal{A}$, it is clear that the square root of $a$ exists in the uniformly bounded case. This can also be obtained directy by combining Lemmas 4.7 and the previous Lemma 6.5.

If $a+\mathbb{1} / n$ is invertible for all $n \in \mathbb{N}$, then $(a+\mathbb{1} / n)^{-1} \in\{a\}^{\prime \prime} \cap \mathcal{A}_{\mathrm{H}}^{+} \cap \mathcal{A}^{\text {bd }}$ for every $n \in \mathbb{N}$ by Lemma 4.6 and $\sqrt{(a+\mathbb{1} / n)^{-1}} \in\left\{(a+\mathbb{1} / n)^{-1}\right\}^{\prime \prime} \cap \mathcal{A}_{\mathrm{H}}^{+}$exists by the first part, and one can thus construct $b_{n}:=(a+\mathbb{1} / n) \sqrt{(a+\mathbb{1} / n)^{-1}}$. By Corollary 4.3, $b_{n} \geq 0$ and one can easily check that even $b_{n} \in\{a\}^{\prime \prime} \cap \mathcal{A}_{\mathrm{H}}^{+}$and $b_{n}^{2}=a+\mathbb{1} / n$. So the previous Lemma 6.5 applies and shows that $\sqrt{a}$ exists.

\section{$7 \quad S u^{*}$-Algebras}

Essentially all of the previous results hold for a class of very well-behaved ordered ${ }^{*}$-algebras:

Theorem 7.1 Let $\mathcal{A}$ be a uniformly complete Archimedean ordered ${ }^{*}$-algebra, then the following additional properties are all equivalent:

i.) All elements of the form $a+\mathrm{i} \mathbb{1}$ and $a-\mathrm{i} \mathbb{1}$ with $a \in \mathcal{A}_{\mathrm{H}}$ are invertible.

ii.) All coercive elements in $\mathcal{A}_{\mathrm{H}}$ are invertible, i.e. $\mathcal{A}$ is symmetric.

iii.) $\mathcal{A}$ is radical and $\sqrt{a}$ exists for all $a \in \mathcal{A}_{\mathrm{H}}^{+}$.

iv.) $\mathcal{A}$ is radical and $|a|$ exists for all $a \in \mathcal{A}_{\mathrm{H}}$.

v.) $\mathcal{A}$ is radical and both $a \vee b$ and $a \wedge b$ exist for all commuting $a, b \in \mathcal{A}_{\mathrm{H}}$, i.e. $\mathcal{A}$ is a $\Phi^{*}$-algebra.

vi.) $\mathcal{A}$ is radical and $a \wedge \mathbb{1}$ exists for all coercive $a \in \mathcal{A}_{\mathrm{H}}^{+}$.

Proof: Implication i.) $\Longrightarrow$ ii.) follows from Corollary 4.12 because $\mathbb{1}+a^{2}=(a+\mathrm{i} \mathbb{1})(a-\mathrm{i} \mathbb{1})$, and for the implication ii.) $\Longrightarrow$ iii.) one uses that every symmetric Archimedean ordered *-algebra is automatically radical by Proposition 4.8 and thus one can apply Proposition 6.6. iii.) $\Longrightarrow$ iv.) is Proposition 6.2, iv.) $\Longrightarrow$ v.) is Corollary 5.7 and v.) $\Longrightarrow$ vi.) is trivial. Finally, for vi.) $\Longrightarrow$ i.), assume that vi.) holds and let $a \in \mathcal{A}_{\mathrm{H}}$ be given. Then $\mathbb{1}+a^{2}$ is coercive and $\left(\mathbb{1}+a^{2}\right) \wedge(n \mathbb{1})=n\left(\left(\left(\mathbb{1}+a^{2}\right) / n\right) \wedge \mathbb{1}\right)$ exists for all $n \in \mathbb{N}$ by Proposition 5.3, so Proposition 5.14 applies and shows that $\mathbb{1}+a^{2}$ has a multiplicative inverse. But then $(a \pm \mathrm{i} \mathbb{1})^{-1}=\left(\mathbb{1}+a^{2}\right)^{-1}(a \mp \mathrm{i} \mathbb{1})$ exists as well.

Definition 7.2 A $\underline{S u^{*} \text {-algebra }}$ is a uniformly complete Archimedean ordered ${ }^{*}$-algebra that has one, hence all of the equivalent additional properties of the above Theorem 7.1 
It is obvious that "Su" refers to "symmetric and uniformly complete". Besides the equivalent characterizations given by Theorem 7.1, a $S u^{*}$-algebra $\mathcal{A}$ also has some other interesting properties:

Because of the existence of square roots, the order on $\mathcal{A}$ is simply the algebraic one, i.e. $\mathcal{A}_{\mathrm{H}}^{+}=\mathcal{A}_{\mathrm{H}}^{++}$, and every unital *-homomorphism $\Psi: \mathcal{A} \rightarrow \mathcal{B}$ into an ordered *-algebra $\mathcal{B}$ is automatically positive, hence continuous for Archimedean $\mathcal{B}$. If $\Psi$ is in addition injective, then it is already an order embedding by Proposition 5.9. Proposition 6.3 shows that the order on $\mathcal{A}_{\mathrm{H}}$ is the unique one with which $\mathcal{A}$ becomes an ordered *-algebra. Unital ${ }^{*}$-homomorphisms between $S u^{*}$-algebras are not only compatible with the algebraic operations and positive, they are also compatible with $\vee$ and $\wedge$, absolute values and square roots by Propositions 5.11 and 6.4. From this point of view, $S u^{*}$-algebras can be seen just as wellbehaved *-algebra, not necessarily as *-algebra with an additional structure, because the order is not subject to any choice and because unital *-homomorphisms between them fulfil all compatibilities one would reasonably expect.

All these properties are typical for $C^{*}$-algebras and complete $\Phi$-algebras, which are important examples of $S u^{*}$-algebras: The uniformly bounded $S u^{*}$-algebras are precisely the $C^{*}$-algebras, because $\|\cdot\|_{\infty}$ in this case is a $C^{*}$-norm by Proposition 3.13, and because conversely, $C^{*}$-algebras carry a natural ordering with respect to which they are well-known to be uniformly bounded and uniformly complete Archimedean ordered *-algebras, and also symmetric due to their well-behaved spectral theory. The commutative $S u^{*}$-algebras are the complexifications of complete $\Phi$-algebras by Proposition 5.8.

As a consequence, the representation theorems for $C^{*}$ - and $\Phi$-algebras partly apply: All uniformly bounded and closed unital *-subalgebras of a $S u^{*}$-algebra $\mathcal{A}$ (especially $\mathcal{A}^{\text {bd }}$ ) are isomorphic to a $C^{*}$-algebra of bounded operators on a Hilbert space. Similarly, all commutative and closed unital ${ }^{*}$-subalgebras of $\mathcal{A}$, that also contain the inverses of all coercive elements (especially bicommutants $S^{\prime \prime}$ of commutative subsets $S \subseteq \mathcal{A}_{\mathrm{H}}$ ), are isomorphic to the complexification of a complete $\Phi$-algebra of continuous functions on a compact Hausdorff space with values in the extended real line by [5]. Similar representation theorems specifically adapted to ordered *-algebras are developped in [15]. With respect to a well-behaved functional calculus we note that already the well-behaved polynomial calculus described in Proposition 4.4 is far from being trivial, but still remains to be extended to a continuous calculus for $S u^{*}$-algebras. Important existing results in this direction are of course the continuous calculus for $C^{*}$-algebras, which also applies to uniformly bounded elements of $S u^{*}$-algebras, and the continuous calculus for $\Phi$-algebras from [2].

\section{$8 S u^{*}$-Algebras of Unbounded Operators}

While $S u^{*}$-algebras are unbounded generalizations of $C^{*}$-algebras and non-commutative generalizations of $\Phi$-algebras, it still remains to give examples of $S u^{*}$-algebras that are not of one of these already wellunderstood types. Clearly, such examples should especially be provided by ${ }^{*}$-algebras of unbounded operators, i.e. by $O^{*}$-algebras like in Example 3.4. In the general case, when $\mathcal{A} \subseteq \mathfrak{L}^{*}(\mathcal{D})$ is an arbitrary $O^{*}$-algebra on a pre-Hilbert space $\mathcal{D}$, it is clear that $\mathcal{A}$ is an Archimedean ordered ${ }^{*}$-algebra. It remains to find sufficient conditions for $\mathcal{A}$ to be symmetric and uniformly complete. Recall that an element $a$ of a ${ }^{*}$-algebra is called normal if $a^{*} a=a a^{*}$.

Definition 8.1 Let $\mathcal{A}$ be a quasi-ordered ${ }^{*}$-algebra. Then a dominant subset of $\mathcal{A}$ is a subset $Q \subseteq \mathcal{A}$ 
of normal and pairwise commuting elements, stable under $\cdot^{*}$, with $\mathbb{1} \in Q$ and such that $q^{*} q$ is coercive and $\lambda q \in Q$ as well as $q r \in Q$ hold for all $\lambda \in[1, \infty[$ and all $q, r \in Q$. For such a dominant subset define the subset $Q^{\downarrow}$ of the commutant of $Q$ in $\mathcal{A}$ as

$$
Q^{\downarrow}:=\left\{a \in Q^{\prime} \mid \exists_{q \in Q}: a^{*} a \lesssim q^{*} q \text { and } a a^{*} \lesssim q^{*} q\right\}
$$

Lemma 8.2 Let $\mathcal{A}$ be a quasi-ordered ${ }^{*}$-algebra and $q, r \in \mathcal{A}$ commuting and with the property that $q^{*} q$ and $r^{*} r$ are coercive. Then $\lambda^{2} q^{*} r^{*} r q$ is coercive for all $\left.\lambda \in\right] 0, \infty[$ and there exists $a \lambda \in[1, \infty[$ such that $q^{*} q+r^{*} r \lesssim \lambda^{2} q^{*} r^{*} r q$ holds.

Proof: Let $\epsilon \in] 0,2]$ be given such that $q^{*} q \gtrsim \epsilon \mathbb{1}$ and $r^{*} r \gtrsim \epsilon \mathbb{1}$, then $\lambda^{2} q^{*} r^{*} r q$ is coercive for all $\lambda \in] 0, \infty\left[\right.$ because $\lambda^{2} q^{*} r^{*} r q \gtrsim \lambda^{2} \epsilon q^{*} q \gtrsim \lambda^{2} \epsilon^{2} \mathbb{1}$. Moreover,

$$
(2 / \epsilon) q^{*} r^{*} r q=q^{*}\left(r^{*} r / \epsilon-\mathbb{1}\right) q+r^{*}\left(q^{*} q / \epsilon-\mathbb{1}\right) r+q^{*} q+r^{*} r \gtrsim q^{*} q+r^{*} r
$$

holds. So $q^{*} q+r^{*} r \lesssim \lambda^{2} q^{*} r^{*} r q$ if one chooses $\lambda:=\sqrt{2 / \epsilon} \in[1, \infty[$.

Proposition 8.3 Let $\mathcal{A}$ be a quasi-ordered ${ }^{*}$-algebra and $Q$ a dominant subset of $\mathcal{A}$, then $Q^{\downarrow}$ is a unital ${ }^{*}$-subalgebra of $Q^{\prime}$, hence of $\mathcal{A}$, and $Q \subseteq Q^{\downarrow}$.

Proof: Clearly $\mathbb{1} \in Q \subseteq Q^{\downarrow}, \lambda a \in Q^{\downarrow}$ for all $\lambda \in \mathbb{C}$ if $a \in Q^{\downarrow}$ and $Q^{\downarrow}$ is stable under * *. Let $a, b \in Q^{\downarrow}$ be given, then there are $q, r \in Q$ that fulfil $a^{*} a \lesssim q^{*} q, a a^{*} \lesssim q^{*} q$ and $b^{*} b \lesssim r^{*} r, b b^{*} \lesssim r^{*} r$. Then $b^{*} a^{*} a b \lesssim b^{*} q^{*} q b=q^{*} b^{*} b q \lesssim q^{*} r^{*} r q$ and similarly also $a b b^{*} a^{*} \lesssim r^{*} q^{*} q r=q^{*} r^{*} r q$ show that $a b \in Q^{\downarrow}$. Moreover, by the previous Lemma 8.2 , there exists a $\lambda \in[1, \infty[$ for which

$$
(a+b)^{*}(a+b) \lesssim(a+b)^{*}(a+b)+(a-b)^{*}(a-b)=2\left(a^{*} a+b^{*} b\right) \lesssim 2\left(q^{*} q+r^{*} r\right) \lesssim 2 \lambda^{2} r^{*} q^{*} q r
$$

and similarly also $(a+b)(a+b)^{*} \lesssim 2 \lambda^{2} r^{*} q^{*} q r$ hold, so $a+b \in Q^{\downarrow}$.

For example, if $\mathcal{D}=\mathfrak{H}$ is a Hilbert space, then $Q:=\{\lambda \mathbb{1} \mid \lambda \in[1, \infty[\}$ is a dominant subset of $\mathfrak{L}^{*}(\mathfrak{H})$ and $Q^{\downarrow}=\mathfrak{L}^{*}(\mathfrak{H})$ is the ${ }^{*}$-algebra of all bounded, i.e. of all $\|\cdot\|$-continuous linear operators on $\mathfrak{H}$. Similar examples generated by a coercive Hermitian, but not necessarily bounded operator in $\mathfrak{L}^{*}(\mathcal{D})$ on a general pre-Hilbert space $\mathcal{D}$ can be constructed analogously. The characterization as a ${ }^{*}$-algebra of continuous adjointable operators carries over as well:

Definition 8.4 Let $\mathcal{D}$ be a pre-Hilbert space and $a \in \mathfrak{L}^{*}(\mathcal{D})_{\mathrm{H}}^{+}$. Then define the positive Hermitian sesquilinear form $\langle\cdot \mid \cdot\rangle_{a}: \mathcal{D} \times \mathcal{D} \rightarrow \mathbb{C}$ as

$$
\langle\xi \mid \eta\rangle_{a}:=\langle\xi \mid a(\eta)\rangle
$$

for all $\xi, \eta \in \mathcal{D}$. The induced seminorm is denoted by $\|\xi\|_{a}:=\sqrt{\langle\xi \mid \xi\rangle_{a}}$ for all $\xi \in \mathcal{D}$.

Recall that the graph topology (see e.g. [11, Def. 2.1.1]) induced by an $O^{*}$-algebra $\mathcal{A} \subseteq \mathfrak{L}^{*}(\mathcal{D})$ on the pre-Hilbert space $\mathcal{D}$ is the locally convex topology defined by all the seminorms $\|\cdot\|_{\mathbb{1}+a^{*} a}$ with $a \in \mathcal{A}$, or equivalently by all the seminorms $\|\cdot\|_{b}$ with $b \in \mathcal{A}_{\mathrm{H}}^{+}$because $b \leq \mathbb{1}+(\mathbb{1}+b)^{2}$ for all $b \in \mathcal{A}_{\mathrm{H}}^{+}$. One 
can check that this set of seminorms $\|\cdot\|_{b}$ with $b \in \mathcal{A}_{\mathrm{H}}^{+}$is even cofinal in the set of all seminorms on $\mathcal{D}$ that are continuous with respect to the graph topology, i.e. for every such continuous seminorm $\mathrm{p}$ there exists a $b \in \mathcal{A}_{\mathrm{H}}^{+}$such that $\mathrm{p} \leq\|\cdot\|_{b}$.

Proposition 8.5 Let $\mathcal{D}$ be a pre-Hilbert space and $Q \subseteq \mathfrak{L}^{*}(\mathcal{D})$ a dominant subset. Then the graph topology induced by the $O^{*}$-algebra $Q^{\downarrow} \subseteq \mathfrak{L}^{*}(\mathcal{D})$ on $\mathcal{D}$ is the locally convex topology defined by all the seminorms $\|\cdot\|_{q^{*} q}$ with $q \in Q$, and the set of seminorms $\|\cdot\|_{q^{*} q}$ with $q \in Q$ is cofinal in the set of all seminorms on $\mathcal{D}$ that are continuous with respect to the graph topology of $Q^{\downarrow}$. Moreover, for all $a \in Q^{\prime}$ the following is equivalent:

i.) $a \in Q^{\downarrow}$,

ii.) $a$ and $a^{*}$ are both continuous as maps from $\mathcal{D}$ with the graph topology of $Q^{\downarrow}$ to $\mathcal{D}$ with the $\|\cdot\|$-topology,

iii.) a and $a^{*}$ are both continuous as maps from $\mathcal{D}$ with the graph topology of $Q^{\downarrow}$ to itself.

Proof: The locally convex topology on $\mathcal{D}$ defined by all the seminorms $\|\cdot\|_{q^{*} q}$ with $q \in Q$ is clearly weaker than the graph topology of $Q^{\downarrow}$. Conversely, given $b \in\left(Q^{\downarrow}\right)_{\mathrm{H}}^{+}$, then also $\mathbb{1}+b \in\left(Q^{\downarrow}\right)_{\mathrm{H}}^{+}$and so there exists a $q \in Q$ such that $(\mathbb{1}+b)^{2} \leq q^{*} q$. Consequently $\|\cdot\|_{b} \leq\|\cdot\|_{(\mathbb{1}+b)^{2}} \leq\|\cdot\|_{q^{*} q}$, and it follows that the locally convex topology on $\mathcal{D}$ defined by all the seminorms $\|\cdot\|_{q^{*} q}$ and the graph topology of $Q^{\downarrow}$ coincide and that the set of seminorms $\|\cdot\|_{q^{*} q}$ with $q \in Q$ is cofinal in the set of all seminorms on $\mathcal{D}$ that are continuous with respect to the graph topology of $Q^{\downarrow}$.

Now let $a \in Q^{\downarrow}$ be given. Then also $a^{*} \in Q^{\downarrow}$ and $\|a(\xi)\|=\|\xi\|_{a^{*} a} \leq\|\xi\|_{\mathbb{1}+a^{*} a}$ and $\left\|a^{*}(\xi)\right\|=$ $\|\xi\|_{a a^{*}} \leq\|\xi\|_{\mathbb{1}+a a^{*}}$ hold for all $\xi \in \mathcal{D}$, which shows that $a$ and $a^{*}$ are both continuous as maps from $\mathcal{D}$ with the graph topology of $Q^{\downarrow}$ to $\mathcal{D}$ with the $\|\cdot\|$-topology, i.e. i.) implies ii.).

Next assume that some $a \in Q^{\prime}$ is continuous as a map from $\mathcal{D}$ with the graph topology of $Q^{\downarrow}$ to $\mathcal{D}$ with the $\|\cdot\|$-topology. Then there is a $q \in Q$ such that $\|a(\xi)\| \leq\|\xi\|_{q^{*} q}$ holds for all $\xi \in \mathcal{D}$, and thus $\|a(\xi)\|_{r^{*} r}=\|r(a(\xi))\|=\|a(r(\xi))\| \leq\|r(\xi)\|_{q^{*} q}=\|\xi\|_{r^{*} q^{*} q r}$ holds for all $\xi \in \mathcal{D}$ and all $r \in Q$ because $a$ and $r$ commute. This shows that $a$ is continuous as a map from $\mathcal{D}$ with the graph topology of $Q^{\downarrow}$ to itself. So ii.) implies iii.).

Finally, assume that $a \in Q^{\prime}$ is such that $a$ and $a^{*}$ are both continuous as maps from $\mathcal{D}$ with the graph topology of $Q^{\downarrow}$ to itself. Then there especially exist $q, r \in Q$ such that $\|a(\xi)\| \leq\|\xi\|_{q^{*} q}=\|q(\xi)\|$ and $\left\|a^{*}(\xi)\right\| \leq\|\xi\|_{r^{*} r}=\|r(\xi)\|$ hold for all $\xi \in \mathcal{D}$, hence $a^{*} a \leq q^{*} q \leq q^{*} q+r^{*} r$ and $a a^{*} \leq r^{*} r \leq q^{*} q+r^{*} r$. By Lemma 8.2, there exists a $\lambda \in\left[1, \infty\left[\right.\right.$ such that $a^{*} a \leq t^{*} t$ and $a a^{*} \leq t^{*} t$ hold for $t:=\lambda q r \in Q$. We conclude that iii.) implies $i$.).

Such dominated *-algebras of operators are especially interesting if they are closed: Recall that an $O^{*}$-algebra $\mathcal{A} \subseteq \mathfrak{L}^{*}(\mathcal{D})$ on a pre-Hilbert space $\mathcal{D}$ is closed if $\mathcal{D}$ is complete with respect to the graph topology of $\mathcal{A}$.

Lemma 8.6 Let $\mathcal{D}$ be a pre-Hilbert space and $\mathcal{A} \subseteq \mathfrak{L}^{*}(\mathcal{D})$ an $O^{*}$-algebra on $\mathcal{D}$, then

$$
\|a\|_{\infty}=\sup _{\xi \in \mathcal{D},\|\xi\|=1}\|a(\xi)\| \in[0, \infty]
$$

holds for all $a \in \mathcal{A}$. 
Proof: The supremum on the right hand side of (8.3) is by definition the minimum of the set of $\lambda \in[0, \infty]$ for which $\|a(\xi)\| \leq \lambda$ holds for all $\xi \in \mathcal{D}$ with $\|\xi\|=1$, or equivalently, for which $a^{*} a \leq \lambda^{2} \mathbb{1}$ holds (where $a^{*} a \leq \infty^{2} \mathbb{1}$ is defined to be always true). By Definition 3.10 and Proposition 3.12, this minimum is just $\|a\|_{\infty}$.

Proposition 8.7 Let $\mathcal{D}$ be a pre-Hilbert space, $Q \subseteq \mathfrak{L}^{*}(\mathcal{D})$ a dominant subset and such that $Q^{\downarrow}$ is a closed $O^{*}$-algebra. Then $Q^{\downarrow}$ is a uniformly complete Archimedean ordered ${ }^{*}$-algebra.

Proof: Let $\left(a_{n}\right)_{n \in \mathbb{N}}$ be a Cauchy sequence in $Q^{\downarrow}$. Then for every $\left.\epsilon \in\right] 0, \infty[$ there exists an $N \in \mathbb{N}$ such that $\left\|a_{n}-a_{N}\right\|_{\infty} \leq \epsilon$ holds for all $n \in \mathbb{N}$ with $n \geq N$, and due to the previous Lemma 8.6, this implies that the estimate $\left\|a_{n}(\xi)-a_{N}(\xi)\right\|_{q^{*} q}=\left\|\left(a_{n}-a_{N}\right)(q(\xi))\right\| \leq \epsilon\|q(\xi)\|$ holds for all $\xi \in \mathcal{D}$, all $q \in Q$ and all $n \in \mathbb{N}$ with $n \geq N$. So for every $\xi \in \mathcal{D}$, this together with Proposition 8.5 shows that $\left(a_{n}(\xi)\right)_{n \in \mathbb{N}}$ is a Cauchy sequence with respect to the graph topology of $Q^{\downarrow}$ on $\mathcal{D}$, and thus converges against a limit $\hat{a}(\xi) \in \mathcal{D}$. The resulting map $\hat{a}: \mathcal{D} \rightarrow \mathcal{D}, \xi \mapsto \hat{a}(\xi)$ is the pointwise limit of the sequence $\left(a_{n}\right)_{n \in \mathbb{N}}$ and is easily seen to be a linear endomorphism of $\mathcal{D}$. The convergence is even uniform in the sense that $\left\|\left(\hat{a}-a_{N}\right)(\xi)\right\| \leq\left\|\left(\hat{a}-a_{n}\right)(\xi)\right\|+\left\|\left(a_{n}-a_{N}\right)(\xi)\right\| \leq 2 \epsilon\|\xi\|$ holds for all $\xi \in \mathcal{D}$ if $n \in \mathbb{N}$ with $n \geq N$ is chosen sufficiently large.

As the ${ }^{*}$-involution is continuous with respect to $\mathrm{d}_{\infty}$ on $Q^{\downarrow}$, also $\left(a_{n}^{*}\right)_{n \in \mathbb{N}}$ is a Cauchy sequence in $Q^{\downarrow}$ and yields a pointwise limit $\tilde{a}: \mathcal{D} \rightarrow \mathcal{D}$. The inner product $\langle\cdot \mid \cdot\rangle: \mathcal{D} \times \mathcal{D} \rightarrow \mathbb{C}$ is $\|\cdot\|-$ continuous as a consequence of the Cauchy Schwarz inequality. Using this it is easily seen that $\tilde{a}$ is the adjoint endomorphism of $\hat{a}$, so $\hat{a} \in \mathfrak{L}^{*}(\mathcal{D})$. The previous Lemma 8.6 together with the above uniform convergence estimate imply that $\hat{a}$ is the limit of the sequence $\left(a_{n}\right)_{n \in \mathbb{N}}$ with respect to $\mathrm{d}_{\infty}$ on $\mathfrak{L}^{*}(\mathcal{D})$.

It only remains to show that $\hat{a} \in Q^{\downarrow}$ : Proposition 3.16 already shows that $\hat{a} \in Q^{\prime}$. Moreover, there exists an $n \in \mathbb{N}$ with $\left\|\hat{a}-a_{n}\right\|_{\infty} \leq 1$, i.e. $\left\|\left(\hat{a}-a_{n}\right)(\xi)\right\| \leq\|\xi\|$ for all $\xi \in \mathcal{D}$, and a $q \in Q$ fulfilling $a_{n}^{*} a_{n} \leq q^{*} q$ and $a_{n} a_{n}^{*} \leq q^{*} q$, i.e. $\left\|a_{n}(\xi)\right\| \leq\|q(\xi)\|=\|\xi\|_{q^{*} q}$ and $\left\|a_{n}^{*}(\xi)\right\| \leq\|\xi\|_{q^{*} q}$ for all $\xi \in \mathcal{D}$. So $\|\hat{a}(\xi)\| \leq\left\|\left(\hat{a}-a_{n}\right)(\xi)\right\|+\left\|a_{n}(\xi)\right\| \leq\|\xi\|+\|\xi\|_{q^{*} q}$ for all $\xi \in \mathcal{D}$, which shows that $\hat{a}$ is continuous as a map from $\mathcal{D}$ with the graph topology of $Q^{\downarrow}$ to $\mathcal{D}$ with the $\|\cdot\|$-topology. The same is also true for $\hat{a}^{*}$, so $\hat{a} \in Q^{\downarrow}$ by Proposition 8.5.

This shows that uniform completeness of certain $O^{*}$-algebras can be guaranteed essentially by a completeness condition on the domain. For the existence of inverses of coercive elements one can then make use of Proposition 4.11:

Theorem 8.8 Let $\mathcal{D}$ be a pre-Hilbert space and $Q \subseteq \mathfrak{L}^{*}(\mathcal{D})$ a dominant subset such that $Q^{\downarrow}$ is a closed $O^{*}$-algebra. Then $Q^{\downarrow}$ is a Su*-algebra if and only if all $q \in Q$ are invertible.

Proof: First assume that $Q^{\downarrow}$ is a $S u^{*}$-algebra and let $q \in Q$ be given. Then $q^{*} q$ is coercive, hence has an inverse, and as $q^{*} q=q q^{*}$ it follows that $q$ is also invertible with $q^{-1}=\left(q^{*} q\right)^{-1} q^{*}$.

Conversely, if all $q \in Q$ are invertible, then also all $q^{*} q$ with $q \in Q$. The previous Proposition 8.7 already shows that $Q^{\downarrow}$ is uniformly complete. Given a coercive $a \in \mathcal{A}_{\mathrm{H}}^{+}$then there exists a $q \in Q$ such that $(\mathbb{1}+a)^{2} \leq q^{*} q$, and then $\mathbb{1} \leq(\mathbb{1}+a)^{2} \leq q^{*} q$ implies $q^{*} q \leq q^{*}\left(q^{*} q\right) q=\left(q^{*} q\right)^{2}$ and therefore $a \leq(\mathbb{1}+a)^{2} \leq q^{*} q \leq\left(q^{*} q\right)^{2}$. It follows from Proposition 4.11 that $a$ is invertible, so $Q^{\downarrow}$ is a $S u^{*}$-algebra. 
Example 8.9 Let $\mathfrak{H}$ be a Hilbert space and $h$ a self-adjoint (not necessarily bounded) operator on $\mathfrak{H}$ which is coercive in the sense that there exists an $\epsilon \in] 0, \infty[$ such that $\langle\xi \mid h(\xi)\rangle \geq \epsilon\langle\xi \mid \xi\rangle$ holds for all vectors $\xi$ in the domain of $h$. Let $\mathcal{D}$ be the dense linear subspace of $\mathfrak{H}$ consisting of all smooth vectors of $h$, i.e. the intersection of the domains of the operators $h^{n}$ for all $n \in \mathbb{N}$, and write $q \in \mathfrak{L}^{*}(\mathcal{D})$ for the endomorphism described by the restriction of $h$ to $\mathcal{D}$, which is coercive in the sense of Definition 4.1. Then $\mathcal{D}$ is complete with respect to the locally convex topology defined by all the seminorms $\|\cdot\|_{q^{2 n}}$ with $n \in \mathbb{N}_{0}$. Moreover, $q$ is invertible in $\mathfrak{L}^{*}(\mathcal{D})$ because the inverse operator $h^{-1} \in \mathfrak{L}^{*}(\mathfrak{H})$ of the self-adjoint coercive $h$ restricts to an endomorphism of $\mathcal{D}$ as well. One can check that the set $Q:=$ $\left\{\lambda q^{n} \mid \lambda \in\left[1, \infty\left[, n \in \mathbb{N}_{0}\right\}\right.\right.$ is a dominant subset of $\mathfrak{L}^{*}(\mathcal{D})$, and as $q$ and hence all elements of $Q$ are invertible, the previous Theorem 8.8 applies and shows that $Q^{\downarrow}$ is a $S u^{*}$-algebra.

One important application of the above Example 8.9 is the case where $h$ is the Hamiltonian operator of a quantum mechanical system. Then the $S u^{*}$-algebra $Q^{\downarrow}$ is essentially the algebra of all symmetries of this system that are bounded by a power of $h$. Note that choosing $h=\mathbb{1}_{\mathfrak{L}^{*}(\mathfrak{H})}$ produces the $C^{*}$-algebra $Q^{\downarrow}=\mathfrak{L}^{*}(\mathfrak{H})$, so the construction of this Example 8.9 is sufficiently general to cover (up to taking suitable *-subalgebras) at least all $C^{*}$-algebras, and clearly many more.

\section{References}

[1] S. J. Bernau and C. B. Huijsmans. Almost f-algebras and d-algebras. In Mathematical Proceedings of the Cambridge Philosophical Society, volume 107, pages 287-308. Cambridge University Press, 1990. 4

[2] G. Buskes, B. de Pagter, and A. van Rooij. Functional calculus on riesz spaces. Indagationes Mathematicae, 2(4):423-436, 1991. 21

[3] G. Buskes and A. van Rooij. Almost f-algebras: Commutativity and the cauchy-schwarz inequality. Positivity, 4(3):227-231, 2000. 4

[4] J. Cimprič. A representation theorem for archimedean quadratic modules on *-rings. Canadian Mathematical Bulletin, 52(1):39-52, 2009. 7, 8

[5] M. Henriksen and D. Johnson. On the structure of a class of archimedean lattice-ordered algebras. Fundamenta Mathematicae, 50(1):73-94, 1961. 2, 4, 21

[6] N. Kouki, M. A. Toumi, and N. Toumi. Commutativity of some archimedean ordered algebras. Positivity, 18(4):805-821, 2014. 4

[7] T. W. Palmer. Banach Algebras and the General Theory of *-Algebras, volume 2 of Encyclopedia of Mathematics and its Applications. Cambridge University Press, 1994. 12

[8] R. T. Powers. Self-adjoint algebras of unbounded operators ii. Transactions of the American Mathematical Society, 187:261-293, 1974. 4

[9] E. Scheffold. ff-banachverbandsalgebren. Mathematische Zeitschrift, 177(2):193-205, 1981. 4 
[10] C. Scheiderer. Positivity and sums of squares: A guide to recent results. In Mihai Putinar and Seth Sullivant, editors, Emerging Applications of Algebraic Geometry, pages 271-324. Springer New York, New York, NY, 2009. 2, 5

[11] K. Schmüdgen. Unbounded Operator Algebras and Representation Theory. Birkhäuser, Basel, 1990. 4, 6, 22

[12] K. Schmüdgen. The Moment Problem. Springer, 2017. 13

[13] K. Schmüdgen. A strict positivstellensatz for the weyl algebra. Mathematische Annalen, 331(4):779-794, 2005. 6

[14] K. Schmüdgen. A strict positivstellensatz for enveloping algebras. Mathematische Zeitschrift, 254(3):641-653, 2006. 6

[15] M. Schötz. Gelfand-Naimark Theorems for Ordered *-Algebras. arXiv e-prints, page arXiv:1906.08752, 2019. 10, 21 